Meta

Journal des traducteurs

Translators' Journal

\title{
Bibliographie de la phraséologie
}

\section{Silvia Pavel}

Volume 40, numéro 4, décembre 1995

URI : https://id.erudit.org/iderudit/003806ar

DOI : https://doi.org/10.7202/003806ar

Aller au sommaire du numéro

Éditeur(s)

Les Presses de l'Université de Montréal

ISSN

0026-0452 (imprimé)

1492-1421 (numérique)

Découvrir la revue

Citer ce document

Pavel, S. (1995). Bibliographie de la phraséologie. Meta, 40(4), 632-653.

https://doi.org/10.7202/003806ar

Ce document est protégé par la loi sur le droit d'auteur. L’utilisation des services d'Érudit (y compris la reproduction) est assujettie à sa politique d'utilisation que vous pouvez consulter en ligne.

https://apropos.erudit.org/fr/usagers/politique-dutilisation/ 


\section{DOCUMENTATION}

\section{Bibliographie de la phraséologie}

La première version de La Bibliographie de la phraséologie (1905-1992) a été publiée en 1993 par le module canadien du Réseau intemational de néologie et de terminologie (Rint), à l'intention des modules membres qui en avaient exprimé l'intérêt lors de la réunion plénière du Comité d'orientation, tenue en juin 1991. Elle constituait l'étape préliminaire des recherches entreprises à la Direction de la terminologie et de la documentation (Bureau de la traduction) en vue de concevoir une méthode d'élaboration de vocabulaires phraséologiques en langue de spécialité. Diffusée en 1994 sous le titre Guide de la recherche phrasésologique en langue de spécialité, cette recherche méthodologique a été accompagnée de deux publications terminologiques à composante phraséologique : le Vocabulaire combinatoire de la CFAO (Conception et fabrication assistées par ordinateur) par Claude Lainé et le Vocabulaire des systemes dynamiques et de l'imagerie fiactale par Silvia Pavel et Monique Boileau. On en trouvera le compte rendu dans les numéros 25-3 et 26-2 de L'Actualité terminologiqué.

Cette nouvelle version comprend des titres parus depuis 1993, mais aussi des références à des ouvrages fondamentaux omis dans la première édition. Je remercie John Humbley du Centre de néologie et terminologie (CNRS. Paris) pour ses commentaires et suggestions en marge de la première édition, et Debora Farji de I'Union Latine (Paris) dont les références transmises ultérieurement sont ici précédées d'un astérisque.

Axée sur la distinction - fondamentale en terminologie - entre langue courante (LC) et langue de spécialité (LS), la recherche bibliographique a été menée à la fois dans des bases de données documentaires comme PASCAL. FRANCIS, ou DIALOG, et dans des publications linguistiques récentes : actes de colloques, périodiques, monographies, thèses et ouvrages lexicographiques. Au cours de nos recherches, nous avons réussi à localiser et à consulter la pluparı des documents mentionnés ci-après. Quelques ouvrages parus au tournant du siècle. les thèses et certains articles publiés dans des périodiques européens, nous sont toutefois restés inaccessibles.

Vu la richesse considérable des études sur la phraséologie LC et leur pertinence pour la réflexion terminologique sur la phraséologie LS, nous avons sélectionné dans la première catégorie les titres relatifs à la formation, à la classification, à la structure et aux fonctions des unités phraséologiques. Dans la deuxième catégorie, ce sont surtout les études consacrées au fonctionnement des unités terminologiques et à leur combinatoire dans le discours spécialisé qui ont retenu notre attention. Cette bibliographie n est dono pas exhaustive mais sélective. 
En plus des références concernant la phraséologie française considérée d'un point de vue unilingue. contrastif ou bien comparatif, il nous a paru utile de porter à la connaissance du lecteur des ouvrages dépassant le domaine français. Certains traitent des universaux du langage en matière d'emplois figurés, de figement lexical, d'idiomaticité, de collocabilité. D'autres analysent les rapports entre la représentation des connaissances et l'évolution des concepts, la néologie et la phraséologie en langue de spécialité. D'autres encore proposent des méthodes de systématisation phraséographique dans les dictionnaires et les bases de données.

Les quelque 400 entrées bibliographiques sont classées dans l'ordre alphabétique par nom d'auteur ou d'éditeur suivi des initiales de leur prénom. Une virgule sépare le nom des co-auteurs et co-éditeurs (voir BURGER, H., BUHOFER, A. et SIALM, A.).

Les titres d'articles apparaissent entre guillemets et précèdent les titres en italique des périodiques dans lesquels ils ont paru. Le volume et le numéro des périodiques sont séparés par un trait. Par exemple, Meta, 36-1, renvoie au volume 36, numéro 1 de cette revue. Lorsque le périodique n'a qu'un numéro de volume, celui-ci est indiqué comme suit : Langages, vol. 63, 1991.

Les titres de monographies, de dictionnaires, de thèses, ou d'actes de colloques sont en italique. Ils sont suivis du lieu de publication, du nom de la maison d'édition et, si possible, du nombre de pages. Par exemple : HANSE, J., DOPPAGNE, A. et BOURGEOIS-GIELEN, H. (1971): Chasse aux helgicismes, Bruxelles, Fondation Charles Plisnier.

L'emploi du caractère gras dans un titre marque la présence d'un élément phraséologique particulier ou d'un mot étranger. Par exemple : ROHRER, CH. (1967) : "Definition of locutions verbales". The French Review. 41-3. pp. 357-367.

ABRAHAM, W. (1975): A Linguisfic Approach (o Mefaphor, Lisse.

AHRENBER(i. L. $(I \varphi(0))$ : «A Crammar Combining Phrase Structure and Field Structurem, COLING- 90 ). Helsinki.

AISENSTADT, E. (1979): "Collocability Restrictions in Dictionaries". Dictionaries and their Users, Papers from the 1978 B.A.A.L. Seminar on Lexicography. Harmann. R.K.K. [ed]. (Exeter Linguistic Studies) University of Exeter, pp. $71-95$

AISENSTADT, E. (1981): “Restricted Collocations in English Lexicology and Lexicography", Review of Applied Linguisfucs, L ouvain. Belgique. vol. 53. pp. 53-61.

AKIM(YT), M. (1981): “An Empirical Investigation of Idiomaticity". Thought Currents in English Literarure. vol. 54, pp. 47-54

ALEXANDER, R.J. (1492) : “Fixed expressions, idioms and phraseology in recent English learner's dictionaries». Euraler '92 Prociedings. Tampere, pp. 35-42.

ALEXFEV, A.A. (1987): "The Development of Verbal Fixed Phrases in Russian", Dissertation Abstracts Imernasional. A. The Humanities and Social Sitences, 47-7. Jan. 2561-A.

ALCiEO, J. (1492) : "The Changing English Vocabulary: Innovation. Survival and Desuetudes", Actes $d u$ $X V^{\circ}$ Comgrès internamonal des linguistés, 9-14 août 1992, Quchec.

ALLEN, S. (1976) : "On Phraseology in Lexicology", (ahiers de lexicologie, 29-2, pp. 8.3-90.

ALLERTON, D. J. ( 1484$)$ : “Three (or four) levels of word cooccurrence restriction», Lingua, NLD, 63-1. pp. 17-40.

ALONCiF: A. (1992): “Machine-readable dictionaries and lexical information on verbs". Euralex 92 Proceredings. Tampere, pp. 195-202.

ALSHAWI. H. (1987) : «Processing Dictionary Definitions with Phrasal Pattem Hierarchies». Compurafional Lingursticis. vol. 1.3.

AMOSSY, R. \& ROSEN, E. (1982) : Les discours du cliché, Société d'Édition d'Enseignement, Paris.

ANICK. P. \& PUSTEJOVSKY, J. (1990) : "An application of lexical semantics to knowledge acquisition from corpora", COLINGi, Helsinki.

ANSCOMBRE, J.-C. (1982) : “(In essai de caractérisation de certaines locutions verbales». Recherches linguisHques, Universite Paris VIII, vol. 20, pp. 5-37.

ANTELMI. D. \& ROVENTINI, A. (|99|): "Semantic Relationships within a Set of Verbal Entries in the Italian Lexical Dillabase", Eurule'. 'S/ Procedings. Benalmadena Malaga.

APRESJAN. Ju.D. (1973) : “Regular Polysemy". Linguissics, 142. The Hague. Mouton. 
ARNAUD. J. ( 1986$)$ : “La comparaison dans les expressions idiomatiques du français littéraire, familier, populaire el argotique: Equivalences allemandes", Lehende Sprachen, Zeitschrift für Fremde Sprachen in Wissenschaft und Praxis, Berlin, 31-4, pp. 16.5-173.

ARNAID. P. \& THOIRON. Ph. (Édit. (199.3): Aspects du vocabulaire, Presses universitaires de Lyon. 199.3. $147 \mathrm{p}$.

ARNTZ, R. (1981): "La Discipline Terminologie dans le cadre de la formation des traducteurs et des interpretes en matiere de langues des specialite.. Theoretical and Methodological Problems of

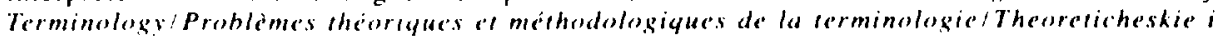
metodelogichesckie vopwowy lerminologii. Münich, Saur, pp. 524-542.

ARNT/., R, (199.3): "Phrasćcologic und (ïhersetzen-liggebnisse und Perspektiven", in Bessé. B. de lédit.l. pp. $575-582$.

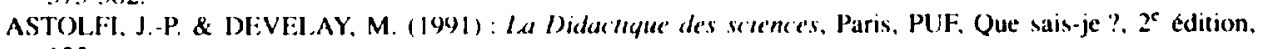
$125 \mathrm{p}$.

ATKINS, B.T.S. \& 7.AMPOL.I. A. leds) (1991) : Compurarional Approaches te the Lexicon, Oxford University Press.

ATTALI. A. e" al (1992) : "Aide au transfert lexical dans une perspective de TAO : expérimentation sur un lexique non terminologique", Mota. Montréal, 37-4, pp. 770)-790.

AUGER, P. \& L.HOMME. M.C. (1994) : "d terminologie selon une approche textuelle : une représentation plus adéquate du lexique dans les langues de specialite", in Kecourek. R. [édit.l, pp. 9.16.

AYMARD-LAPAIU, N (1982): "Du concept ì la langue de specialite : Essai d'organisation doun voca-

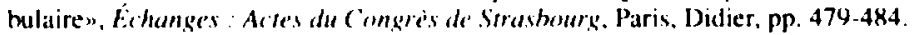

*BAI.BASTRE I FERRER, J. (1977): Dicoromart de modismes a frase's fore's, 11 volumes, Barcelona, Portic.

BALLY. Ch. (1905): Prérit de strfistique. Cienive. Eggimann.

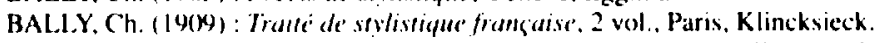

BAN, F. (1987): "I es unites lexicales de plusieurs mols sous laspect de la linguistique contrastive et de

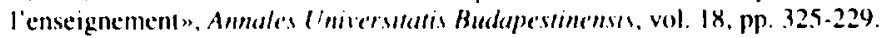

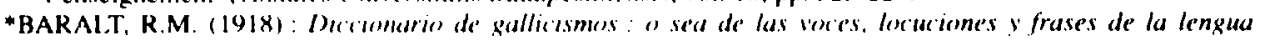
franceses que so han intresductedo, Madrid, Imp. Graf. Excelsior.

BARDOSI. V. (198.3): "I.a rédaction d'un dictionnaire onomasiologique de lecutions : exquisse d'une proble-

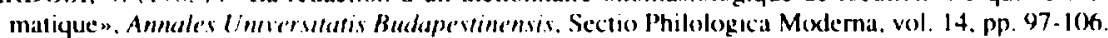

BARDOSI. V. (198.5): "Les limites de l'utilisation des dictionnaires de locutions", Annales Umicersifatrs Buclupestinc'nsis. Sectio l.inguistica. vol. 13. pp. 17-26.

BARDOSI, V. \& CSINK. I. (1986): “Le trattement des lecutions idiomatiques par micro-sordinateur", Area

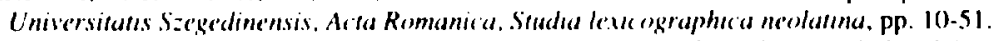

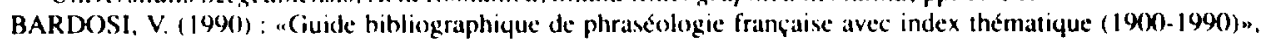
linguisucas Ime'sngatmome's, 14-2. pp. 349-402.

BAR-HILLEL, Y. (1955): "Idioms". Marhme Translatiom of Languages, Locke, W.N. \& Boxth, A.D. [eds]. New York-london, pp, $18.3-193$.

BAUER, L. ( $(980)$ : "Deux problèmes au sujet des noms composés comprenant un premier élément verbal en français molerne". Le Framgass Maderne. vol. 48. pp. 219-225.

BAUSCH, K.-R. (1963): lerbum und verbale Periphrase im Fransösischen und ihre Transposition im Englischen. De'uss he'n und Sirantsithen. (Thèse), 'Tübingen.

BAISC 11, K.-R. (197.3) : “Expressions défigees im Franfosischen”. Pravis de's ne'usprachliche'n Unterrichrs. 20-2. pp. 144-195:20-3,p. 3014.

BECKER, J.D. (1975): "The Phrasal lexicon", Proceedengs of the Workshep on Theoretical lssues in NLP. P. 701.73.

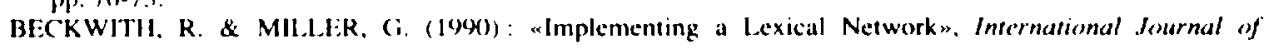
le'vcosstuph: 3-4, pp. 302-312.

BFJOINT. H. (1993) : “L a Défintion en terninologie», in Amaud. P. \& Thoiron. Ph. lédit.l. pp. 19-26.

BEJOINI. H. \& THOIRON, Ph. (1993) : "Macrosiructure et microstructure dans un dictionnaire de collocations en langue de upécialité", in Bessé. B. de [édit. J. pp. 51.3-522.

BEL ANGI:R. A. 11992 ) : "Syntaxe des groupe' prédicatifi Nom de Nom". Ace's du Xl' ('ongrés international de's linguriste's. Quéhec, 9.14 aloût 1902.

BENES, $:$ : (19XI): “D formale Siruktur der wissenschaftlichen Fachsprachen in syntaktischer Hansiche", in Bungarten. T. Wissenschafisspras he, München, Fink, pp. 185-212.

BENNETT, T.J.A. (|981) : "Tramslating Colour Collocitoons", Mefo, 26-3, pp. 272-280.

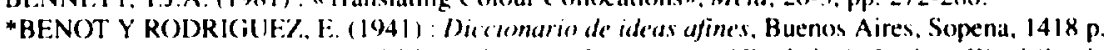

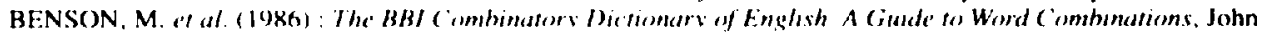
Benjamins.

BENSON. M. (I489) : " The Structure of the Collocational Dictionary". Internatuomal Journal of Lexigagraphy, 2-1.pp. 1-14. 
BENVENISTE. É. (1966) : «Formes nouvelles de composition nominale», Bulletin de la Sociéré de linguistique de Paris. ol-1.

BENVENISTE. É. (1966) : Prohlèmes de linguistique générale, 2 vol., Paris, Gallimard.

BENVENISTE. E. (1969) : Le Vocuhulaire des institusions indo-europeennes, 2 vol.. Paris, Minuit.

BERARD-DUGOURD, A. \& RICHARD, G. (1986): “Le traitement des locutions dans l'analyse du langage naturel”. (Étude $n^{\circ}+101$, Centre Scientifique IBM France).

BERNARD, (i. (1974) : “Les locutions verbales françaises". La linguisrique, 10-2, pp. 5-17

BERNET, $($. ( $(1989)$ : : Le nom propre dans la phrasécologie et le lexique : I'exemple du mot Amérique el ses dérivés." Actes du congrès international de linguistique et philologie romane. Tréves (1986). Tübingen. Max Niemeyer, vol. 4. pp. 520-529.

BESSÉ. B. de ( $|\varphi 9|)$ : "Le contexte terminggraphique», Meta. Montréal, 36-1. pp. 111-120.

BESSÉ. B. de [édit.] (1993) : Phraséologie ćt termunotogie en traduction el en interpréfation, Actes du colloque international de (ieneve, octobre 1991 , Traduction et terminologie. $2 / 3$ Luxembourg, CCE, 588 p.

BJORKMAN, S. (1978): “Le type avoir besoin. Étude sur la coalescence verbo-nominale en français», Uppsala, Acta Universitaris Upsaliensis. (Studia Romanica Upsaliensis, 21 ).

BLAMPAIN, D. (1993) : "Traduction et écosystèmes terminologiques", in Bessé. B de [édit.].

BLANCHON, E. (1991): "Choisir un logiciel de terminologie". La Banque des mots, numéro spécial 4. pp. 5 - 58 .

BLINKENBERG, A. (1960) : Le Probleme de la transitisité en fransais merderne, Munksgaard. 366 p.

BLONDELLLE. F. (1980) : Mémento de svnthese du verhe fransais. Comment dire: les locutions françaises inme ées par l' infinisif pour s'exprimer correctemem, Paris. Blondelle.

BLOY, L. (197.3) : Eirégèse des lierux communs, Paris, Mercure de France.

BOCQUET, ( ( 199.3$)$ : “Phraséologie et traduction dans les langues de specialite», in Besse, B. de [edit.]. pp. 271.284

BOILFAU, M. (199.3) : “Terminologie el phraséologie : un heureux mariage", L'Actualité rerminologigue. 26-2. pp. 16-17

BOLINGER. D. (1971): The Phrasal Verh in English, Cambridge, Mass., Harvard University Press. 187 p.

"BOLO(XNE. J.C. (1989) : Les grandes allusions. Dictionnaire commenté des expressions d'origine littéraire, Paris, Larousse.

BONHOMME. M. (1992) : "Sémantique de la métonymie et théories des cas", Actes du XV" Congrès internanonal des linguistés, Québec, 9-14 août 1992.

BORGMANN, D. A. (1986) : aFrom Rags to Riches ... and Beyond". Word Ways : The Journal of Recreational linguistucs, Morristown. NJ. 19-1. pp. 57-6.3.

BORIL.I.O. A. (1971): „Remarques sur les verbes symétriques français», Langue française, vol. 11.

BOUCHARD. C.. STEFANO. (i. di \& MC GILLIVRAY, G. (1985) : "La locution : probleme de traduction*. Actes du colleque international La Locution. Montreal, 1984. Moyen Fransais (Le), 14-15. pp. 19-27.

BREMOND, C. (1985) : "Concept et thème", Poétique, no 64, pp. 41.5-423.

BRESSON. D. (1989) : “La distribution du sens dans les locutions à verbe support”. Travaur du Cercle linguis. Rique d'Ala-en.Provence. $n^{\circ}$ 7. pp. 57.72

BRISCOE. T. \& COPESTAKE. A. (1991): "Sense extensions as lexical rules", Computational Approaches to non-literal language : metaphor, metonymy, idiom, speech acts. implicature. Proceedings of IJCAI Work shop. Sydney, pp. 12-20.

BROCK. S. \& LEVY, L.L. (1988): Kdiom's Delight: Fascinating Phrases and Linguistic Eccentricities : Spanish. French. Llatian. Lasin. New York. Times Books, $158 \mathrm{p}$.

BR(XKE-ROSE. Ch. (1965) : A (irammar of Metaphor, London, Mercury Books. 343 p.

*BROWNE, V. Dizionario di false analogie ef ambigue affinita francese. inglese e italiano, Zanichelli, 272 p.

BRINOT, F, (1965): La Pensée el la langue, Paris, Masson. $3^{\mathrm{C}}$ ed., $9.54 \mathrm{p}$.

BUDIN. (). ( $14(0))$ : "Terminological Analysis of L.SP Phraseology". Terminology Science and Research, IITF, 1-1/2, pp. 64-69.

BUDIN. G. \& GALINSKI. Ch. (199.3): “Ühersetzungsorientierte Phraseologieverwaltung in Terminologie datenbanken». in Bessé. B. de (édit.). pp. 565-574.

BÜHLFR. H. (199.3) : “Of Terms and Texts", in Besse. B. de lédit.], pp. 423-430.

BUJAS. \%. (1975): "Testing the performance of a bilingual dictionary on topical current texts». Studia Romanica et Anglica Zagrahiensia. 39, pp. 193-204.

BURGER. H., BUHOFER, A. \& SIALM. A. (1982): Handhuch der Phraseologie. (avec la collaboration de Brigit Eriksson. Jürg Häusermann, Angelika L.inke. Thomas Scherer el Blanche Schweizer). Berlin, New York.

BURGER, H. \& JAKSCHE. H. (197.3) : “Idiomatik des Deutschen *. Tubingen Germanistiche Arheitshefte, 16.

BURIDANT, C. (1989): “LApproche diachronique en phraséologie : Quelques aspects de l'ancien et du moyen français... Traveaur de linguistique ef de Philologie. Strashourg. vol. 27, pp. 127-149. 
CALZOLARI, N. (1984): "Detecting Patterns in a Lexical Database». Procedings of the $10^{\text {m }}$ International COLING, Stanford, Calif.

CALZOLARI, N. (1991): "Acquiring and Representing Semantic Information in a Lexical Knowledge Base". Proceeding. of the Workshop on Learcal Semantics, Pustejovsky, J. [ed]. Berkeley. Calif.

*CAMARGO. S. \& STEINBERG. M. (1987) : Dicionario de expressores idiomaticas metaforicas inglês. pormugues. Mc Graw-Hill, Sáo Paulo.

CANDEI. D. (1984): "Ambiguité d'origine polysémique dans une langue de spécialité", Cahiers de Lexicolegree, 45-2. pp. 21-.32.

CANDEL, D. (1994): "Sur l'introduction de mols nouveaux dans les contextes spécialisésm. Rhetoric and Solvesfus Tieday, NBHGi, Peter Lang, pp. $71-74$.

CANDEL. D. |eddit.) (1994) : Français scientifique et fer hnique ef dicriomnare de langue. CNRS-INaLF, Paris, Didier Érudition, $221 \mathrm{p}$.

CANDEL. D. (1994) : "Vers un dictionnaire du françars scientifique el technique", in Candel, D. (édit.). pp. $185-201$

CANDEL, D. \& LAFON, P. (1994) : "Approche lexicale des registres en langues de spécialité, Mera. 39.4, pp. 807-815.

CARADEC. F. (1977) : Dicrionnaire du frangais argotique et popularre, Paris, Larousse.

*CARBONELL, S. (1983, 1986): Dizionario fraseologico completo italiano-spagnolo el spagnolo-italiano. 2 volumes. Milano. Ulricho Hoelpi.

*CARNEADO MORE, Z.V. (1985) : “Notas sobre les variantes fraseologicas". Annuario L/L, no 16, pp. 269-277.

*CAROLL. E.J. (1946) : The Yachtsman's Glossary of nautical terms and expressions (en 5 langues), London, Simpkin Marshall.

CARVALHO, J.G.H. (1986): "Verbes et locutions causatifs en portugais en comparaison avec le français". Congres international de linguistique et philologie romanes. 17, 1983, Aix-en-Provence/Université de Provence, Marseille, J. Lafitte. vol. 4. pp. 325-334.

CASAGRANDE, J. (1975): "Fossilization in French Syntax", Janua linguarum, Series practica. vol. 207. pp. 23-35.

CATHERINE. R. ( 1988) : “Anthologie de mots reçus", Banque des Mots, vol. 36, pp. 129-153.

CAUSSE. J.-P. (1978): Dictionnaire des vrais amis. Dicrionnaire fransais-anglais de locutions similaires placée's dans un comeneve'. Bériers.

CELLARD. J. \& REY. A. ( 980() : Dictionnaire du français non conventionnel, Paris, Hachette, $89.5 \mathrm{p}$.

CHABRIDON. J. \& L.ERAT, P. (199.3) : “Terme et famille de termes". La Banque des Mots, no 5, pp. 55-63.

CHAFE. W. (1968): "Idiomaticity as an Anomaly in the Chomskyan Paradigm». Fonndarions of Language. vol. 4-2, pp. I(K)- 127.

*CHARBONNIER, E. (1991) : La momnate de singe ou comme'me recomnairre les expressions aswes du MoyernAge. Paris, Hatter.

CHARLES, W. \& MILLER, G.A. (1989) : "Contexts of Antonymous Adjectives". Applied Psycholinguistics. vol. 10. pp. $357-375$

CHÉTRIT, J. (1978): “Les composés nominaux à joncteur a. I. Etude lexicologique», Cahiers de lexicologie. $32-1, \mathrm{pp} .65-81$.

CHETRIT, J. (1978): «les composés nominaux a joncteur à. 2. Étude lexicologique, sémantique el syntaxique". (cuhiers de lexirohigie, 33-2. pp. 53-70.

CHETRIT, J. (1979) : “l és composés nominaux a joncteur à. 3. Étude lexicologique, sémantique el syntaxique (suite) (caheress de lesicologie. 35-2, pp. 91-10.5.

CHOUEKA. Y. (1988) : "Looking for a needle in a haystack, or locating interesting collocational expressions in large textual databases”, RIAO'88 ('omferene Premededings, MIT. Cambridge. Mass. pp. 6019-623.

CHOUL. J.C. (198(b) : "Si muove, ma non troppos : An inquiry into the non-metaphorical status of idioms and phrases", Scmuiste's, pp. 89.98.

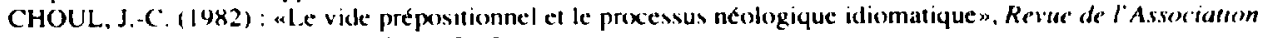
aushiconse de lingusustique. 2-2, pp. $21-30$.

CHOU/L. J.-C. (1982): “Rigles d'interpretation idiomatiquen, Journal of the Allantic Preninces Linguistic

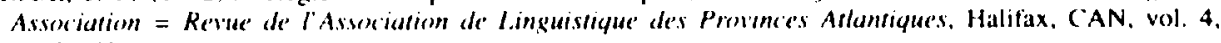
pp. 36-53.

CHOUL. J.C. (1987): "Sémantique de la locution". Rerue Queberoise de lingustique Thérique et Appliquéc. Trois-Rivières. (anada. 6-3. pp. 117-1.30.

CHURCH, K. \& HANKS, P. $(1990)$ : "Word association norms, mutual information and lexicography". Computational Linguistics. 16-3.

CLAFYS. P. F. (1989): "Theoretical and translational aspects of phraseology (microform]". These (M.A.) Iniversity of New Brunswick. Ottawa. Bihliotheque nationale du Canada.

CLAS. A. (1994) : “collocations et langues de upecialité". Mofa. 39-4. pp. 576-580. 
(LAS. A. \& SEUTIN, E. (1989) : J'parle en tarmes. Dictionnaire de lixutions et d'expressions figurés au Quéher. Montréal, Sodilis.

CLAUSEN. (I. \& L.YLY. E. (1994): "Criteria for Identifying and Representing Idioms on a Phraseological Dictionarym, in Martin, W. et al. ledsl, pp. 258-262.

COHEN, B. (1986) : Lexique des coocurrents, Bourse - Conjoncture économique, Montreal, Linguatech.

COHEN, B. (1993): “Méthodes de repérage et de classement des cooccurrents lexicaux», in Bessé. B. de lédit.], pp. $505-512$

COLSON, J.-P. (199.3): «Ébauche d'une didactique des expressions idiomatiques en langue érangere», in Bessé, B. de lédit.l. pp. 16.5-I R(I.

('OP. M. (199()): "The Function of Collocations in the Dictionary", Buclalex '8\& Proceedings, $3^{e}$ Congres Furalex. Akadémiai Kiado. Budapest, pp. 35-46.

('ORMIER. M. (1989): "La terminologie: du terme au texte". Terminologie diachronique, Paris, CILF, pp. 212-2IX.

-CORNUBERT, R. (1970): Dichonnatre chimique allemand-fransais: mofs et locusions frequemment rencontréex dans les textes de langue allemande. Paris, Dunod.

COSERIU, E. (1966) : «SIructure lexicale et enseignement du vocabulaire», Ac'es du premier colloque internarional de linguisfique appliquée. Nancy, 26-31 (xtobre 1964. Faculté des Lettres et des Sciences Humaines de l'Universite de Nancy. (Annales de 1'Est, Memoire 31), pp. 175-217.

COSERIL. E. (1967) : "Lexikalische Solıdaritaten". Poefica, Zetischrift fur Sprach-und Literaturwissenschaft. vol. 1. pp. 29.3-303.

COSERIL, E. (1968): a Lex structures lexématıquesm, Probleme der Semantik, 3-16, Wiesbaden, Steiner.

COULON. B. (1983): Deutsche und franzïsische idiomatische Redewendungen. Untermitarbeiter von Philippe Coulon. Munich, Hueber, $152 \mathrm{p}$.

COURTNEY, R. ( I983) : The I Angman Dicionary of Phrasal Verhs, Harlow. Longman, 734 p

COUTIER, M. (1494) : "Tropes et termes : le vocabulaire de la dégustation du vinm. Meta, 39-4, pp. 662-675.

COWIE: A.P. $(1981)$ : :The Treatment of Colloxations and Idioms in Learner's Dictionariesm, Applied Lingutstics, 2-3, pp. 223-23.5.

COWIF. A.P. (1986) : "Collocational dictionaries - a comparative viewn, Proceedings of the $4^{\text {th }}$ Joint AngloSonuer Semenar on English Studies, London, pp. $61-69$

"COWIE, A.P. \& MACKIN, R. (1975, 1983): Oxford Dic tienary of Current Idiomaric English. 2 vol., London, ()xford Univ. Press.

CULIOLI, A. (198I): «Sur le concept de notionm, BULAC; no 8, pp. 62-79

(URAT, H. (1982): La locution verbale en fransais moderne. Essai dexplication psycho-systematique, Les Presses de I’Université Laval, 319 p.

(URAT. H. (1985) : “La relation privilégiée entre l'agent et l'objet dans les locutions verbales», La locution. Acte's du colloque internatmonal. Universite McGill. Montreal, 15-16 octobre 1984, Le Moyen Frangais, vol. 14-15, pp. 28-5.5.

("AP. H. \& (iALINSKI, C. leds| (1987): TKE' 87 : Terminology and Know/edge Engineering. Frankfur, Indeks Ferlag.

(YAP. H. \& NEDOBITY, W. leds| (1990): TKE '90): Terminology and Knowledge Engineering. 2 vol. Frankfurt, Indeks Ferlag.

DANL.OS, 1.. (1981) : "l a morphosyntaxe des expressions figees", Langages, vol. 63, pp. 53-74

[DANI.OS, 1.. lédit.] ( 1988 ) : "l es Expressions figées", langages, n" spécial, vol. 90.

I)ANI.OS, L. (198X) : antroduction : lexique-grammaire des expressions figées», Langages, vol. 90, pp. 5-6.

IDANLOS. L. (1988) : aLes Phrases à verte support etre Prép". Lansages, Paris, vol. 90, pp. 23-37.

DARMESTETTER. A. (1979): La lie de's mots étudiée dans leurs significations (1887). Paris, Ed. Champ Libre.

DEBILI, F. (1982): Analyse syntaxico-semantique fondse sur une acquisition automatique de relation. lex/cales-simantiques. Thèse de doctorat. Université de Paris XI. France.

DE CARRICO, J. \& NATTINGER, J.R. (I98K) : al exical phrases for the comprehension of academic lectures". The ESP Jorrmol. Washington DC: vol. 2.

DECARY, M. \& LAPALME. (;. (1490) : "An Fditor for the Explanatory and Combinatory Dictionary of Contemporary French (DEC (C)", ('omputational Lingutstics, vol. 16, pp. 145-154.

DEESE, J. (1962) : Form Class and the Determinants of Association", Journal of Verbal Learning and Verbal Behavior. vol. 1. pp. 79-84.

DFL.ABRE. M. (1984) : ales deux types de comparaisons avec commen, Le frangais moderne, 52-1/2, pp. $22-47$

DEMERS, (i. ( $\mid(92)$ : “Caractéristiques des sujets el des verbes dans les textes scientifiquesm, Actes du $X V$ conurès international des Inguistes, Quebec. 
DESCAMPS. J.-L. (1985) : "Quelques principes pour l'analyse méthodique des contextes des mots en langues de spécialitén. Bulletin de la Commissison Interuniversiture Suisse de Linguistique Appliquée. Neuchâtel. Suisse, vol. 41. pp. 96-110.

DESCAMPS. J.-L. (1994) : "Pour qui élathorer un dictionnaire de langue techno-scientifique ?", in Candel, D |Édit...pp. 15|-|83.

DESCAMPS, J.-L.. (1994) : “Tournoi pour l'accommodement des dictionnaires de collocations", Mera, 39-4, Pp. 561.575.

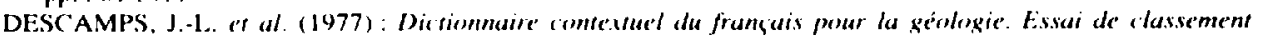

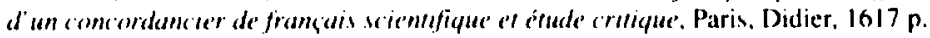

DESCAMPS. J.-L. ot al. (1492): Dictommaire contedud du français polutuque, 11 microfiches, in Descamps, J.P. â al lédit.l.

DESCAMPS. J.-1. of al lédit.] (1992) : Simantique of romourdanness, Coll. Saint-Cloud, CNRS-INal.F, diff. Klincksieck.

"DESMET, 1. (1991) : “Terminologia e fraseologia : Iendencias actuais”, Terminologias, 3-4, Listora, Termip. pp. 1(1)-30.

DIAZ. O. (1983, 1984) : "Observations sur les expressions lexicaliseesm. Cahiers du Centre Interdisciplinaire des Sciences du langage, vol. 5, pp. 139-15.3

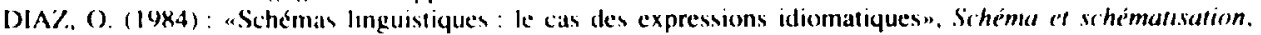
vol. 20. pp. 87-94.

DIAZ, (). (1986): “Partır du bon pneu : L.'Expressıon idiomatique à travers l'expression publicitare / Jdiomatic

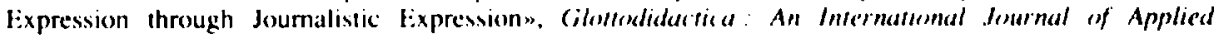
Linglussics, Poenan. Pologne, vol. 18. pp. 75-82.

DIAZ., (). (1986) : "liexpresion idhomallique dans le dictionnaire : probleme de didaxie culturelle». Cahiers du

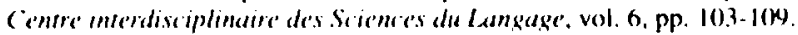

DOBROVOI:SKIJ, D. (|488) : "Phraseologie als Objekt der Universalienlinguistik", Leipgig. VEB Verlag Enzzklopidstic.

DOBROVOL:SKIJ, D. (1(x)2): "Phraseological Universals: Theoretucal and Applied Aspects". Meaning and (irammar. ('rosss-lingusstic Perspertives, Kefer, M. \& van der Auwera, J. |eds|. Berlin / New York. Mouton de (iruyter.

DOBROVOL:SKIJ, D. (1994): «ldioms in a Semantic Network: Towards a New Dictonary Type". in W. Martin e'l al. leds|. pp. 26.3-270.

DOSTIE, (i., MFL CUUK. I. \& POL.GUERE. A. (1992) : "Méthodologie d'élaboration des entrées lexicales du Dictuonnaire Explicatif et Combinatoire du Français Contemporain". International donenul of Lexicography. 5-3. Oxford IIniv. Press. pp. 165-109.

DRASKAl, J., LAUREN, (Ch. \& NORIMAN, M. ledsl (1989): "The Trojan Horse, or the Terminological Ambush: A Pragmatic Demonstration of the Importance of the Correct Handling of L.SP Elements in I.GP

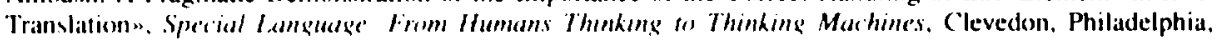
pp. $384-396$.

DRASKAI. J.K. (1991): "Some Reflections on Rquivalence/Aquivalenz as a Tern and a Concept in the Theory of Translation". Me'ta. Montréal, 36-1.

DUBOIS. M.-M. BRIIIIER, Y. HIENRIOT-(iRUNDY. E., SURIVET, S. \& MAHAFFEY. D. (1973): Dichemnare francais-anglais de loc ations of expressoms verbales. Paris, Larousse.

DU(iAS. A. \& DI SCIULL.O. A.-M. (1985) : "Le rôle des déterminants dans les expressions figées de langues

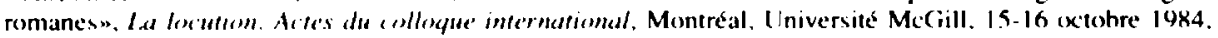
le moven franciais, vol. 14-1.5. pp. 56-60\%.

DUGAS. A. \& DI S(III.L. ). A.-M. (I98.5): «l.e traitement lexical des phrases figés du français, de

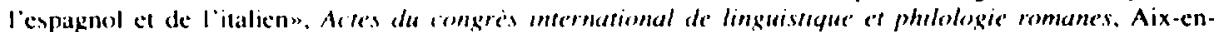
Provence. Universitte de Provence-Marscille. Laffitte. 1. 3. pp. 279-292.

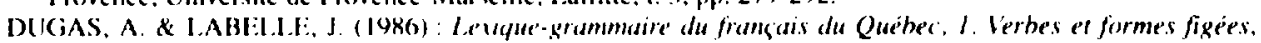
Département de linguistique. I nnversité du Québece a Montréal, Kapport de recherche CRSH 410-85-1382.

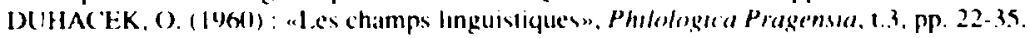

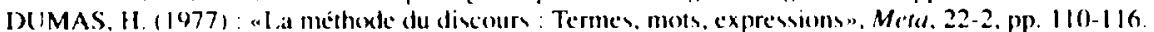

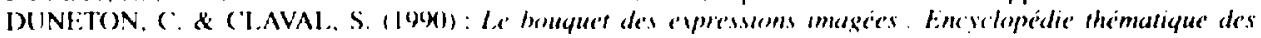

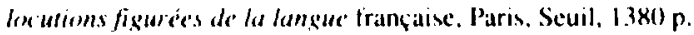

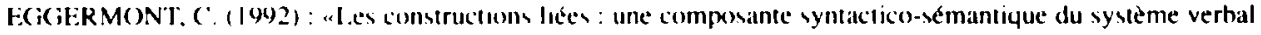
françailis». ITl. 95-96. pp. $29-47$.

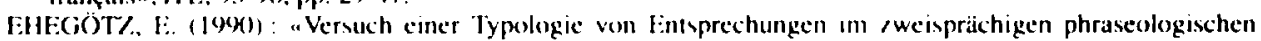

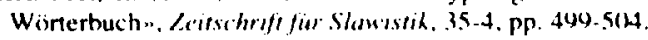

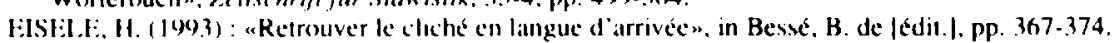

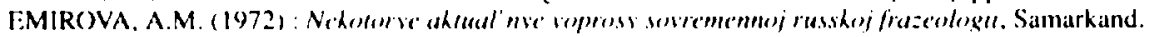

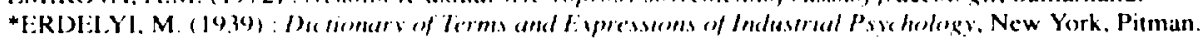


ERVIN-TRIPP, S. (1970) : "Substitution, Context, and Association», Norms of Werd Association, Postman. L.. Keppel, G. ledsl, New York. Academic Press.

ETTINGER, S. ( $(99)$ : "Idiomatik und Sprachpraxis. Zu einen Buch von Vilmos Bardosi, De Fil en aiguille». Lehende Sprar hen. 3-25, pp. 1.31-1.32

EVVENS, M. W. [ed| (|(x)2) : Relutional Models of the Lexicon, Cambridge University Press. 1992.

FELLbA(IM, Ch. (I94)) : "English Verbs as a Semantic Net". Imernarional Journal of Lexicography. 3-4. pp. $278-301$.

FElLBAUM. ('h. (1492): "A Relational Network of English Verbsm. Terminology \& Documentation in Spectalized c'ommunicutum Procecedings. Ottawa. Department of the Secretary of State of Canada, pp. 170-187.

FELL.BALIM. (h. (1992) : “Coxccurrence and Antonymy", CLSC Reporr No 52, Cognitive Science Laboratory. Princeton (Iniversity.

FERNANIX), C. (1978) : “Towards a Definition of Idiom : Its Nature and Function». Studies in Language, 2-3. pp. $31.3-34.3$.

FERNANDO). (h. \& Fl.AVELL. R. (1981): “On Idiom : Critical Views and Perspectives”, Exeter Linguisfic Studies, vol. 3, (Iniv, of IExeter, GRB, $94 \mathrm{p}$

FIALA. P. (1487): "Pour une approxhe discursive de la phraséologie : remarques en vrac sur la locutionalite et quelques points de vue qui s'y rappontent, sans doute", Langage et société, vol. 42, pp. 27-44.

FIALA. P. (1989): "Figements et phrasélogie : état des recherches actuelles". Courants sociolinguistiques

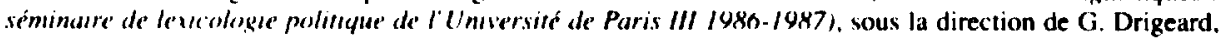
P. Fiala, M. Toumier. Parıs, Klincksieck, pp. 149-155.

FILLENHALIM, S. \& JONES, L. V. (1965): "Grammatical Contingencies in Word Association», Journal of Verbal lecarning and leerbal Be havior, vol. 4, pp. 248-255.

FII.LMORE, (h. J. ( 1468$)$ : "The Case for Case", Universals in Linguissic Theory. New York. Holt Rinehan \& Winston, pp. $1-\mathrm{ks}$.

FILI.MORF, (h. J. (1984): "Remarks on Contrastive pragmatics". Contrastive Linguistics. Prospects and Prohlems. Fisiak. J. led]. Moulon.

FILLMORE, Ch. J. (1992): “(Corpus linguistics vs. Computer-Aided Armchair Linguisticsm, Directions in Corpus L.enguisfics, Berlin. Mouton de Gruyter, pp. 35-60).

FILLMORE, (h. J. ( 1994$)$ : "Lexicography and Ethnographic Semanticsm, Plenary Lecture. EURALEX 94 Proceredings. Amsterdam.

FILLMORE, ( $\mathrm{h}$. J. \& ATKINS. B.T.S. (1994) : «Staring where the dictionaries stop; the challenge for computational lexicography", Compurational Approaches so the Lexicon. Atkins, B.T. \& 7ampoli, A. [eds]. Oxford University Press.

FIL.LMORE, (h. J.. KAY, P. \& O'CONNOR. N.C. (1988): "Regularity and Idiomaticity in Grammatical Constructions: The Case of Let Alonem, Language, 64-3, pp. 501-538.

FIRZLAFF: B. \& HAENELT, K. (1992): «Applying Text Linguistic Principles to Modelling Meaning Paraphrasesm, Euralex '92 Procesdings, Tampere, pp. 21.3-220.

Fl.AUBERT, (i. (191.3): Le Dic tomnaire des udées reçues, Paris, Conrad.

FlEISCHER, W. (1482): „Phraseologie der deutschen Gegenwartssprache», Leipzig, VEB Bibliographisches Instritur.

FONAGY, 1. (1982) : Situation el significarion, Amsterdam-Philadelphia, John Benjamins.

FONAG;Y, I. (198.3) : "Des cliches politiques en tant que modèle d'érosion sémantique». From sounds to words. essays in homor of Claes-Christian Elert. Karl-Hampus Dahlstedt. Hansson. A. el al. [eds], Umea Studies in the Humanities. Acta Universitatis Umensis, 60), Stockholm, pp. 107-114.

FONTANIER. P. (1968) : Les Figures du discours (1830), Paris, Flammarion, rédit., 507 p.

FONTENFLL.E. Th. (1992): "Collocation acquisition from a corpus or from a dictionary : a comparison*. Euralex 92 Procededings, Tampere, pp. 221-228.

FONTENFLLE, Th. (1994) : al/sing Lexical Functions to Discover Metaphorw, in Martin, W. ef al. [eds]. pp. $271-278$.

FONTENELLE. Th. el at (1994) : “Survey of Collocation Exıraction Tools», Project DECIDE (MLAP 93-19). D-1a. Université de Liège.

"FONTES. L.E. FIERREIRA (1482) : Gilossarto de termos e expressoes de cienctas do solo, Universidade Federal de Viçosil.

FRADIN. B. ( 1988) : “Decrire un verbem, Lexique. vol. 6. pp. 97.138

*FRAENCKEL. B.B. (1987): (Glossario inglês-português de termos médicos: termos médicos, termos correlatos. expressoes idiomatics. 35(X) verhetes, BB. Fraenckel, Rio de Janeiro.

"FRANCE, H. (1990) : Dir Hennaire de la lungue verse ar haismes, nénlogismes, focutions étrangeres, patois. Nigel (iauvin. $361 \mathrm{p}$.

FRECKLETON, P. (1985): Une comparaison des expressions de l'anglais el du frangais, (These de $3^{c}$ cycle. Université Paris 7. LADL.), Paris 
FREI. H. (1953) : Le livre des de'ux mille phrases. Sociéte de Publications romanes et françaises, 40, Geneve.

FUGGER. B. (1984) : “La Planification linguistique en France contemporaine : Langue commune et langue de specialité : Le Nouveau Vocabulaire de la médecine". Socrolinguisrique' des langues romanes. Aix-enProvence. Presses (Iniv. de Provence. pp. 105-117

GAATONE. D. (1981) : "Les lecutons verbales : pour quoi faire?". Rerue romane. 16-1/2. pp. 49-73.

GAATONE. D. (1982): "Locutions et catégories linguistiques". Graser linguistische Studien. vol. 16. pp. 44-51.

GAATONE, D. (1984) : "La locution ou le poids de la diachronie dans la synchronie", Le moyen français. vol. 14/15. pp. 70-81.

GALINSKI. Ch. (1990) : "Terminology and Phraseology". Trrminology Science and Research, 1-1/2, pp. $70-86$.

GALISSON. R. (1981): Imentare themastique et sintugmatique du francans fondamental. Paris, Hachetie.

GALISSON, R. (1983) : «Pour une méthodologie de l'accès aux locutions figuratives en français, langue maternelle et etrangère", De's mots pour communtquer, Paris. Cle Internationale, pp. 87-159.

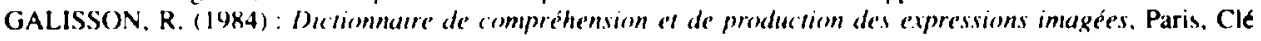
Internationale.

GALISSON. R. (1984): Les Mors mode demplon: les expressions imagées, Paris. Clé internationale. (CARAMEL).

GAMBIER, Y. (1993) : «Socioterminologie et phraséologie», in Bessé. B. de [édit.]. pp. 397.410)

GARY-PRIEUR. M.-N. (198.5): De la grammaire à la linguistique' : l'étude de la phrase. Collection Linguistique, Paris, Armand Colın, $165 \mathrm{p}$.

GAUDIN, F. et al. (1993) : "Sémantique et terminologie : sens et contextes», in Bessé, B. de [édit.]. pp. $411-422$.

GEBRUERS, R. (1994): “Tournures: Where Valency and Idiomaticity meet". International Journal of Lexicography. 7-2. pp. 142-157.

GEERAERTS, D. (1994) : "Varieties of Lexical Variation", in Marin. W. el al. leds]. pp. 78.83.

GENETTE, G. ( 1960$)$ : "La Rhétorique restreinte". Communic ations, vol. 16, pp. 158-171.

GENTILHOMME. Y. (1968): "Les ensembles flous en linguistique". Cahiers de linguistique théorique et appliquér. S, Bucarest, pp. 47-65.

GENTILHOMME. Y. (1984) : “Les faces cachées du discours scientifique». Langue française. 64. pp. 29-37.

GENTIL.HOMME. Y. (1992): "Ll.éclatement du signifié dans les discours techno-scientifiques", Signifiant. référent. réel. CsRLE. 20. Annales Littéraires de l'Université de Besançon. pp. 29-66.

GENTILHOMME-KOUTYRINE. Y. (1994) : "Regard sur la terminologisation en lexicologie». Mesa. 39-4. pp. $546-560)$

GENTNER, D. \& (iENTNER, D. (198.3) : "Flowing Water or Teeming Crowds : Mental Models of Electricity". in Cientner, D. \& Stevens, A. leds], Mental Mode/s, Hullsdale, N.J.

GEORGE. K.E.M. (1970) : "Expressions figurees empruntees au jargon du tisserand". Revue de linguistique romarnc, 34-13.5/136, pp. 395-402.

GERBER, B.L. \& STORZER, (G.H. (1976): Dictionary of Modern Fiench Jdioms, New York, Garland, 2 vols, $1228 \mathrm{p}$.

GIRY, J. (1974) : «Syntaxe et lexique : un exemple de classe sémantique”, Re'vue romane', vol. 9, pp. 57-68.

GIRY-SCHNEIDER, J. (1978): "Les nominalisations en français: loperateur faire dans le lexique". Langues et cultures, vol. 9, (ieneve, Dros.

GIRY-SCHNEIDER, J. (1984): "Jean fait le (généreux + diable): Constructions productives et expressions

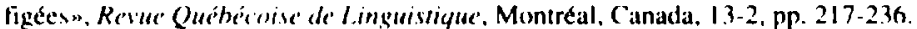

GIRY-SCHNIEIDER. J. (1986): "Les noms construits avec faire: compléments ou prédicats?". Langue francuise. vol. 69, pp. 49-5.3.

GIRY-SCHNEIDER, J. (1987): “Les prédicats nominaux en français. Les phrases simples à verbe suppon». Langue's 't C ultures, Cienève.

GLAAP, A.R. \& WFLLER, F.-R. (1979): "Auswahlbibliographic /ur ldiomatik im Fremdsprachenunterricht" (Englisch-Franziosisch), Die Neueren Sprachen, vol. 78, pp. 586-595.

(iL.̈̈SER, R. (1982): "The Stylistic (ompenent of Languages for Special Purposes». Fachsprache

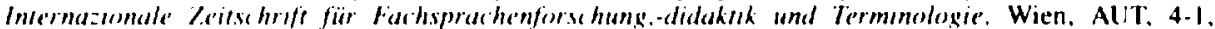
pp. $2-11$

(iL.ASER, R. (1484): "The Translation Aspect of Phaseological Units in English and German». Papers and Studies in Comtrassive Linguissirs. Poznan, POL., vol. 18. pp. 123-134.

GLÄSER. R. (1987): "The concept of LSP Rhetoric in the Framework of Modern Text Linguisticsm. Philologeca Pragensias, CSK, 30-5, pp. 113-119

GLÄSER, R. (1994) : "Relations Between Phratseology and Termonology in Specialised Language with Special Reference to I:nglish", in Koxourek, R. |Eddit. |, pp. 41-60). 
GLÄSER. R.. HULLEN, W. \& SCHULZE. R. |eds] (1988) : "The Grading of Idiomaticity as a Presupposition for a Taxonomy of Idioms". Meaning. Sense and World Knowledge in Lexical Semantic. Tubingen. Niemeyer.

GLEITMAN. L. (1990) : "The Structural Sources of Verb Meanings", Language Acquisirion, vol. 1. pp. 3-55.

GOETSCHALCKX, J. (1973) : "Essai d'étude comparative des locutions et termes d'un glossaire plurilingue". Mrla. $\mid$ |K-1/2, pp. 26|-267.

GOETSCHALCKX. J. (1493) : "Terminologie et phraséologie ", in Bessé. B. de [édit.]. pp. 477-484

GOFFIN, R. (1972) : «Structures lexicales. terminologies techniques et glossaires contextuels multilingues» Metu. IX-1/2, pp. 237-253.

GOFFIN, R. (1993) : "Du synthème au phrasécolexème en terminologie différentielle», in Besse, B. de [Gdit.]. pp. $431 \cdot 438$.

*GOMEZ. L.L. (1987): Dicionario de expressoses idiomaricas americanas, Livraria Pioneira Editora. Sto Paulo.

GOUADEC, D. (1989): «Traduction. rédaction, (franc)isation", Traduction, Terminologie, Rédaction, UQTR - Université Concordia, Montréal, 2-1, pp. $51-58$.

GOUADEC, I), (199.3): "Corrélations entre phraséologie et teminologie dans la constitution de banques de données", in Bessé, B. de [édit.], pp. 549.564 .

CiOtIADEC, D. (1993): "Extraction, description, gestion et exploitation des entités phraséologiques*, Terminologies noutelles, I0, Bruxelles, Rint, pp. 8.3-91.

CiOUADEC, D. (édit. ( 1993 ) : Terminologie el Phraséologie. Acteurs el aménageurs. Actes de la $2^{c}$ universita d'automne en terminologie, Rennes 20-25 septembre, Paris. La Maison du Dictionnaire, 1994, 298 p.

GOUGENHEIM. (j. (1971) : Erude sur les périphrases verbales de la langue francaise. Paris, Nizet.

GOUGENHEIM, (i. (1971): "Une catégorie lexico-grammaticale : les locutions verbales", Erudes de linguisrique appliquere, vol. 2. pp. St-64

GOLGENHEIM, (i. (1975) : "le vocabulaire des expressions figes", Les Mots francais dans l' histoire et dan: la vite. vol. 3, pp. 16-20.

GRADY, M. (1971): "An English Idiom : Subject - Object -Object - Actor", Linguisfics, vol. 73, pp. 44-45.

GRAUBER(i, W. (1989) : «Proverbs and Idioms: Mirrors of National Experience'? $m$. Lexicoxraphers and thei, Works, Exeter Linguistic Studies. (BBR, vol. 14, pp. 94-49.

(iRÉCIANO. (i, (19K4) : aPour un apprentissage des unités phrasélologiques». Nouveaux cahiers d'allemand. vol. 2. pp. $95-113$.

(iRECIANO, (i. (1986): «Actualités phrasélogiques», Verbum, 9-3, pp. 319-340.

(iRECIANO, (i. (1986) : “Determinants el idiomes, Determinants : syntaxe et sémantique”, Recherches lingaistiques, Centre d'Analyse Syntaxique, Université de Metz, Faculté des Lettres et Sciences Humaines, vol 11. pp. 73-86.

GRÉ(IANO, G. (1986): al.es inférences de l'idiomem. Travaux de linguisfique el de litsérature. 24-1, pp. 1.39-153.

CRÉCIANO. ( . (1986): “les operations enfouies du codage idiomatique». Recherches anglaises ef oméri(aines (RANAM), vol. 19. pp. 179-194.

GRECIANO, G. lédit.] (1989) : Furophras' 8R. Phraséologie Contrastive, Collection Recherches Germaniques, Strastourg. Université des Sciences Humaines, Deparnement d'Études Allemandes, vol. 2.

(iRÉCIANO. (i. ( 199,3$)$ : "Priorités phraséographiques pour l'allemand el le français», in Bessé. B. de [edit.]. pp. $345-35 K$.

(iRFCIANO. G. (199.3): "Vers une modélisation phrastologique: Acquis et projets d'EUROPHRAS» Termunologies nouvelle's, 10. Bruxelles, Rint. pp. 16-22.

(iRFCIANO, G. (1995) : “Phraseologismus und Nicht-Phraseologismus. Zur Abgrenzung zwischen festen und freien Wortfügungenn, dans P. Valentin (Ed) Saat und Emte, Mélanges ufferts d̀ Jean Fourquet pohi son Sheme ammiversarre. Paris-Sorbonne. Linguistica Palatina Colloxua.

(j.R.E.C.O. (1980-1994): Decuments pour l'histoire du vorahulaire scientifique. CNRS-INaLF, Paris, 14 bl.

GREFENSTETTE, (;. (1994) : “Corpus-Derived First. Second and Third-Order Word Affinities», in Marti ; W. et al. ledsl. pp. 279-290.

GREFENSTETTE. (i. \& TELHFL. S. (1995): "Corpus-based Method for Automatic Identification of Suppon Verbs for Norminalizations". Proceedings of the 7 th Conference of the European (hapter of the Assocision for Computatronal Limpuisfics, Dublin.

GRICE. H.P. (1989) : Sucheses in the Way of Words, Cambridge, Mass, Harvard Univ. Press.

GRIFFIN, J. \& ENRICiHT, D.J. leds] (198.5) : "Euphemisms in Greece and Romew. Fair of Speech: The Us's of fiuphemism, Oxtord. $222 \mathrm{p}$.

CROSS, (i. (19X8) : "Depré de figement des noms composés", Langages, vol. 90, pp. 57-72.

GROSS. G. (1989) : Les constructuons comerses du fransars, Geneve-Paris, Droz.

(iROSS. M. (1981) : «Les bases empiriques de la notion de prédical sémantiquem, Langages, vol. 63, pp. 7-?.,

(iROSS. M. (1984) : "Les noms traceurs". Cahiers de lexicologie, 44-1. pp. 105-1 36. 
GROSS. M. (1984) : «Une Classification des phrases ngées du français". De la syntaxe à la pragmatique. Amsterdam, Benjamins, $388 \mathrm{p}$.

GROSS. M. (1985) : “Sur les détermmants dans les expressions figees”, Langages, vol. 79, pp. 89-117.

GROSS, M. (1986) : “Les Nominalisations d'expressions figés", Langue Fransalsc, vol. 69, pp. 64-84.

GROSS. M. (1988) : "Sur les phrases tigées complexes du français", Languc fransais', vol. 77, pp. 47-70)

GROSS. M. (19K8) : "l ces limites de la phrase figée”, langages. vol, 90, pp. 7-22.

GROSS. M. (199()) : (irammaire lransformanommelle du fransais. Sintaxe de ladierbe. Evry. CERIL.

GROSS. D. \& MILLER. K.J. (199()) : "Adjectives in WordNet". Internatermal Jourmal of [exexicography. 3-4. pp. $265-277$.

GUILBERT, 1. (1971): “De la formation des unités lexicales", (irand Larmasse' de la langue françuisc. Paris. Larousse, vol. I. pp. $[X-X X X]$.

GUILBERT, L. (1975): Ia irsativifé levicale, Paris, Larousse, $285 \mathrm{p}$.

GUIL.LEMIN-FI.ES(HER, J. (1991): «Recurrent Pattems in Language and Translation". Terminologie' " Truduc tom, (IEE: Luxembourg, n"3, pp. 4.5-54

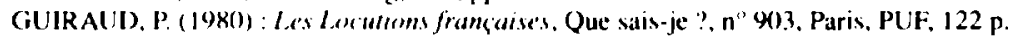

GUMPEL, L. (1974) : "The Siructure of Idioms: A Phenomenolocial Approach». Semustica, 12-1, pp. 1-40.

(iÜNTHFR. K. $(\mid 990)$ : “Dictionary of Phraseological Terms. Wioncrhuch phraseologischer Termini”, Linguistische Studien. Reihe A : Arbeitsterichte, n" 20.5. 3-164.

1.AENELT, K. et al. (1991): "The Textual Development of Non-Stereotypic Concepts". Procecdings of the

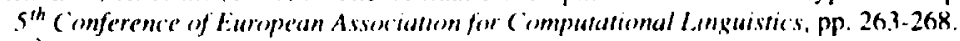

iAGECF, Cl. (1992): "Towards a sexio-operative conception of linguistics", Actes de XV Comprès internarional de's lenguisises, Québec.

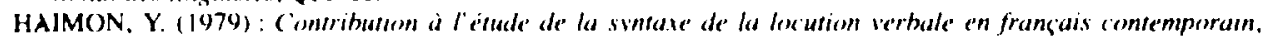
Mémoire de maîtrise, Université de Tel Aviv.

HAMILTON. 1. (1993): "Phraseology in Translation at the United Nations: some Examples”, in Besse, B. de Edit.l. pp. 24.5-251).

HANSE. J., DOPPACINE, A. \& BOURGiEOIS-GilELEN. H. (1971): (hasse am belgicismes. Bruxelles. Fondation Charles Plisnier.

HANSE. J.. DOPPAGINE. A. \& BOURGIEOIS-GIELEN, H. (1981): Nourelle rhasse aux helgicismes, Rruxelles. Fondation (harles Plisnier.

HARTMANN, R.R.K. (1994): "The Ise of Parallel Text Corpora in the (ieneration of Translation Equivalents for Bilinguall Lexicography", in Martin, W. et al. [eds]. pp. 291-297.

HAUSMANN, F.J. (1979): "In dictionnaire des collocations est-il possible ?". Tratam de linguisuque of de listirurure. 17-1, Pp. 187-195.

HALISMANN, I.J. (1984): "Wortschat/lemen ist Kollokationslemen. Zum Lehren und Lernen franzosischer Wotverbindungen". Praxs des newsprachliche'n Unterrichss. vol. 31. pp. 395-406.

HALIS !ANN, F.J. (1985): "Kollokationen im deutschen Wörterbuch. Ein Beitrag aur Theorie des lexiko-

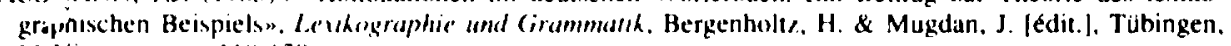
Mi Niemeyer, pp. 118-129.

HAUSMANN, FJ. (1989): "le dictionnaire de collocations", Dirfionedres. Dictionnares. Wörterhürher. Internatonal Encyclopeda of Lenuography, Hausman. F.J. et al lédit.l. Berlin. New York. de Gruyter, pp. $1010-1019$

HEII). (1. (149.3) : "Decrire les collexations : deux approches lexicographiques et leur application dans un outil informatisé ", in Bessé, B. de lédit. J. pp. 523-548.

HEII). 11 (1994): “On Ways Work Work Together Topics in lexical Combinatorics», in Marin, W. ef al. led , pp. 226-257.

HEII: (. (1944): "Relating Lexicon and Corpus: Computational Support for Corpus-Based Lexicon Building in DEI.IS $\cdots$ in Martin. W. of al leds]. pp. 459-47l.

HEID 1. (1995) : "Using Lexical Functions for the Extractoon of (ollocations from Dictionaries and Corpora". in Wanner. 1. . [ed].

HEII) I) \& FREIB()TT, (i. (1991): “Colloxations dans une base de donnés terminologiques et lexicalesm. Miru, 36-1, pp. $71-91$

HEIT. U1. \& RAAB, S. (I989) : “Colloxatoons in Multilingual Cieneration". Procededings of the fih Conference"

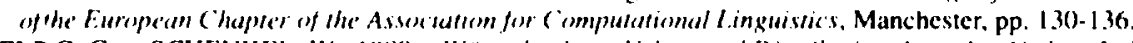

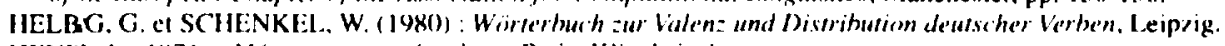

HENPI, A. (1971) : Métemsme el mitophere', Paris, Klincksicck.

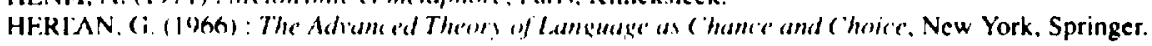

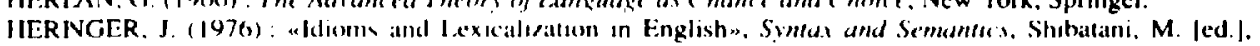
vo. 6. New York, Suantord \& London. Academic Press, pp. 2015-2l6.

HER;CHBER(i-PII:RR()T. A. 119801 : "Prohlématique du cliché. Sur Flaubert". Poćlique. vol. 43, ij. $3.34-34.5$ 
HERSLUND, M. \& SORENSEN, F. (1992) : "Verb Valency and Automated Text Processing”, Computational Approaches wo Tex Understanding. pp. 105-113

HERTING, B. (| $99(0)$; : "Transformations lexico-pragmatiques dans la traduction : leur nécessite, justification, leurs causes et effets pour le texte traduit", Babel, 36-3, pp. 129-142.

HEYLEN. D.L. of al $(1991)$ : "Collocations and the Lexicalisation of Semantic Operationsw, EUROTRA, Ulirecht.

HEYLEN. D. \& MAXWEL.L., K. (1994) : "Lexical Functions and the Translation of Collocationsw. in Marin. W. el al | |eds|. pp. $298-305$.

HCXKETT, C.F. (1956) : "Idiom Formation". For Roman Jakobson, Halle, M. el al. [eds]. The Hague. Mouton. pp. 222-229.

HOFFMAN, L. (1982) : «Terminology and LSPw, Terminologies for the Eighties, Munich, Saur, pp. 391-402.

HOIINHOL.D. I. (1993): “Terminologisch relevante Phraseologie in Fachtexten», in Besse. B. de [Edit.]. pp. $251-270$.

HOHNHOLD. I. \& SCHNEIDER. T. (|99|): «Terminological Records and Lexicon Entries. A Contrastive Analysism, Me/a. 36-1.pp. 161-173.

HORNE, (h. (1992): "La métaphore: quelques définitions contemporaines». ALFA, vol. 5. Halifax. pp. $179-180$.

HOUGAARD, C. (1986) : “Danish and slavic phraseology contributions to an analysis of idiomatics on a contrastive hasis". Papers and Sindies in Comerastive Linguistics, vol. 21, pp. 145-196.

HUHER. H. (1985) : “Dites-le avec des fleurs ! À propos des euphemismes de la langue courantem, Praxis des neusprac hlic hen Unterrichts, 32-3. pp. 260)-265.

HUHER, H. (1985): "Les Metaphores du haut en has de l'echelle sociale et professionnellew, Moderne Sprachen : Organ der Verhundes des Österreichischen Neuphilologen fur Moderne Sprachen. Literatur, und Pa. Vienne. 29-3/4, pp. 33-43.

HUBER. H. \& HOUILLON, A. (1988): "Les Pièges qui guettent le traducteur de citations». lehende Sprachen. 3.3-1, pp. 26-29.

HUGUET. F. 11933) : le langage figuri au seizieme siècle. Paris. Hachette.

HUL.TENBFR(i. H. (19)3) : L' renforcement du sens des adjectifs et des adverhes dans les langues romanes. Uppsala. (These).

HUMBERT. J. (1954) : Le fransais idiomarique. Neuchatel.

HUMBLEY, J. (1993): “Exploitation d'un vocabulaire combinatoire: syntaxe, phrastologie, analyse conceptuellew, Terminologies nouvelles, $\mathrm{n}^{\circ}$ 10, pp. 95-102.

HUMBI.EY, J. (1994): "L.exploitation des bases de donnees terminologiques pour la lexicographie specialiste', in Candel, D. |édit.J. pp. 37-51

HUMS. L. (1971) : “Terminologie und Phraseologie - ihr Verhältınis zueinander auf dem Hintergnund der russichen Terminologie des Eisenhahnbaus". Wissenschaftliche Zeitschrift der Technischen Universität Dresden. 5-20. pp. 1251-1254

HUMS, L. (197K): «Phrasecologie und Terminologie in ihren Wechselbezichung*. Sprache in Wissenschaft und Terknik. Lin Summelhund. Hoffmans, L. [ed], Leipzig. pp. 104-116.

IL.G. G. (1985): „Expressions», Me/a, 30-1. pp. 65-67.

IRUJO. S. (1986): "Don'। Put Your Leg in your Mouth : Transfer in the Acquisition of Idioms in a Second Language ", TESOL Quarter/v, Cedar Falls, 20-2, pp. 287-304.

ISATCHENKO, A. V. ( 1948$)$ : "Morphologie, syntaxe et phrastologie», Cahiers de Ferdinand de Saussure. vol. $7, \mathrm{pp} .17-32$

IVIR, V. (1988): "Collocations in Dictionaries, Monolingual and Bilingual», Lexicographical and Linguistic Studies. Essays in honour of (G.W. Turner, Burton. Tl et al. leds]. Cambridge, New Hampshire, pp. 43-50.

JACOBI. D. (1994): "Lexique et reformulation intradiscursive dans les documents de vulgarisation scientifique", in (andel, D. [édit.]. pp. 6.3-75

JEAY, M. (1985) : "l es Évangiles des quenouilles: de la croyance populaire à la locutionw, La locution. Actes du colloque international. Université McGill, Montréal. 15-16 octobre 1984. Le moyen français, vol. 14-15. pp. $2 \times 2-301$.

JOCHNOWIT\%. G. (1986): «Acceptable but Not Grammatical». Maledicta: The International Journal of Verhal Askression. Waukesha, Wi, vol. 9. pp. 71-74.

JOHNSON. M. (1987): The Body in the Mind. The Bodily Basis of Reason and Imagination, Chicago, London.

JOHNSON, M. (|\$92): "Philosophical Implications of Cognitive Semantics». Cognitive Linguistics. 3-4. Mouton de Gruyter. pp. 345-366.

JOLY. H. (1988) : “De francophonia», Banque des Mots, vol. 36, pp. 123-128.

J1)RGE. (i. (199.3) : “Les expressions idiomatiques correspondantes : analyse comparative», in Besse. B. de [édit.].

JUSTESON, J.S. \& KATZ. S.M. (1991): :Redefining antonymy: the textual structure of a semantic relation*. Proceedengs of the Seventh Annual Conference of the UW Centre for the New OED. Waterloo, Canada. 
KASSAI, G. (1974) : “Syntagmes figés et attirance entre lexèmes». Études contrastives sur le françuis et le hongrois, Studia Romanica Universitatis Debreceniensis. Ser. Ling., fasc. III. pp. 23-37.

KASSAI, G. (1983) : “Traduction, centralité, marginalité", Linguistique (La), Paris, 19-2, pp. 117-127.

KASSAI, (i. (199) ): "Pour un dictionnaire des connotations”. Actes du I R. Colloque international de linguis. tique fonctionnelle. Prague.

KATZ. J. (1973): "Compositionality. Idiomaticity and Lexical Substitution", A Festschrifi for Morris Halle. Anderson. S.R. \& Kiparsky, P. leds], New York. Holt, Rinehart and Winston, pp. 357-376.

KAUSSEN, K. J. (1987): "Interlingual Transfer in the Translation of Scientific and Technical Texts", Desserfation Ahstracts Inte'matumal, Ann Arbor. MI, 4k-5.

KAYSER, D. (1993) : “La représentation des connalssancesm. ('ourrier du (NRS, ${ }^{\circ}$ RO).

KETTRIDGE, J. O. (1939) : French ldoms and figurative phrases with many quotations. New York.

*KETTRIDGE., J.O. (1972) : Dictiomnaire franças-anglasis. anglais-français de termes et locutions seshniques

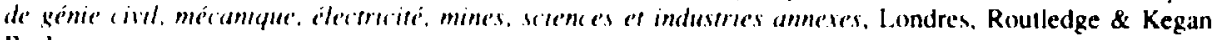
Paul.

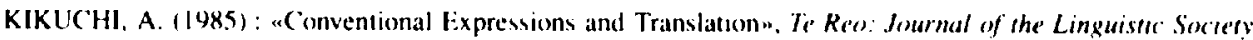
of Ne'w Zeuland. Auckland. New Zeialand, vol. 28, pp. 61-79.

KIPFER, B. A. (1987): "Dictionaries and the Intermediate Student: Communicative Needs and the Development of User Reference Skillsm, in Cowte. A.P. fed) The Dictionary and the Language Learner. Tühingen, Niemeyer.

KIRK-(iREENE, (C.W. (1986): "French dioms: some comparisons, contrasts and catches", Modern langewage's, 67-3, pp. 142-14.3

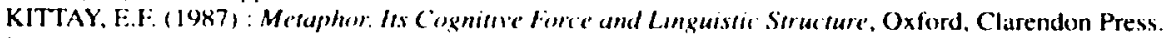

KJAER. A.L. ( $\mid(99(1)$ : "Context-conditioned word combinations in legal language", Terminology science and rescurch. 1-1/2.pp. 21-32.

KJAER. A. L. (1990) : «Phraseology Research. State of the Art”, Terminology Science and Research. JITF, 11/2. pp. $3-20$

KJAER. A.L. (1987) : «Zur Darbietung von Phraseologismen in einsprachigen Wönerbüchern des Deutschen aus der Sicht ausländischer Textproduzenten", Bciträge zur allgemicinen und germanistischen Phraseolegieforschumg. Korhonen. J. Jédit.J. Intemationales Symposium in Oulu, 13-15 Juin 1986. Oulu, pp. $165-181$.

KJELLMER. G. (1984): “Some Thoughts on Collocational Distinctiveness in Recent Developments in the Use of Computer Corporat in English L.anguage Research", Costerus. NLD, vol. 45, pp. 16.3-171.

KL.ARE. J. (1989) : «le statut des phraséolexemes dans le cadre d une lexicologie et d'une lexicographie

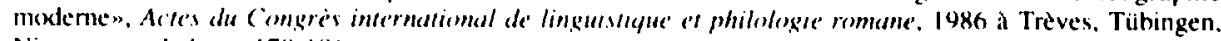
Niemeyer. vol. 4. pp. 178-186.

KLEIBER, (3. (1986): "Le problème des phrases habituelles: une altemative aux approxhes quantificationnelles, la solutoon ontolognque de (i.N. Carlson". Situdica romanica posnamiensia, vol. 12, pp. 11-26.

KNOWLES, F. (199.3) : "Dictionaries for Advanced l.earners and Users of Foreign Languages". Verhatim. $19-3$.

KNOWI.FS, F. \& ROF: P. (1994): “Facilitating the Corpus-Building Process and Maximising the Analytical Field : A LSP-Oriented Case Studym. ('OMPlEX 94 Proceedings, Kiefer, F.G. \& Pajzs, J. ledsl, Budapest. Hungarian Academy of Sciences.

KNOWLES. F, \& ROE. P. (1994): "LSP and the Notoon of Distrubution as a Basis for Lexicography", in Martin. W. af al. leds]. pp. 3(K-319.

KOCOUREK R. (1941): La langue française de la fechnique d" de la sichence, Wiesbaden. Oskar Brandstetter Verlag, $2^{C}$ édition.

KOCOUREK. R. (1992): "La formation lexicale mélaphorique en terminologie". Actes de XV"Comprès

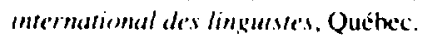

KOCOUREK. R. (1492): "Specialized Texts and Terminology as Ohjects of Linguistic and Pragmatic

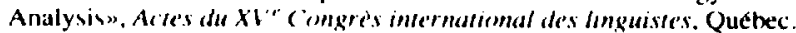

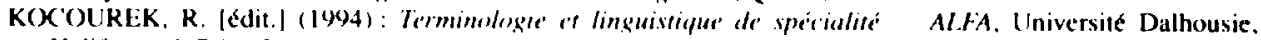
Halifax, vol. $7 / 8,516 \mathrm{p}$.

KOCOUREK, K. (1904) : "Set Metaphors in English Linguistic Terminology", in Kocourek. R. [édit.]. pp. $361-394$.

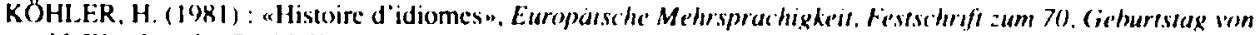
M. Wisndroszka. Pookl. W. [edtut.). Tübingen, Niemeyer. pp. Its 27.4

KRAHI.. ( $:$ (1944): "Aspokle des Kumbunationswissens von Verben und Substantiven im Vergleich zu Adjektiven am Beispie englischer Temperaturlexeme», in Martin, W. et al leds]. pp. 320-324.

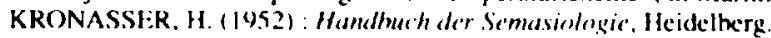


KROUMOVA, 1. (1971): “Comparaisons siéréotypées dans la langue française, formées à l'aide de la conjonction comme (essai daperçu général, de classification et d'analyse)». Trudove na Velikotarnovskia Unversitet, Kiril i Metodii. Filologitcheski Fakultet. Sofia, vol. 9. 1971/1972, pp. 193-216.

KUCERA, A., CLAS, A. \& BAIIDOT, J. ( 1991$)$ : Dictionnairo compact des sciences ef de la technique, 2 vol. (allemand-français, français-allemand). Wiesbaden, Oskar Brandstetter Verlag, 1991 el 1995.

KUKUL.SKA-HUL.MF. A. (199)) : «Un dictionnaire actions-acteurs pour l'informatiquew, Terminogramme. n 5. pp. $21-24$

LABELL.E. J. (1984) : "Le prédicat nominal avec support avolr. Contribution a l'élude de la phrase simple». Lexique-grammaire des langues romanes. Acte's du premier colloque europden sur la grammaire el le levique comparés des langues romanes, Paleme. 1981. Guillet. A. \& La Fauci. N. [édit.]. (Linguisticae Investigationes Supplementa. 9). Amsterdam-Philadelphia. pp. 165-198.

LABELLE: J. (1985): "Les expressions figées en français du Québec studi canadesi, Bari, Schena Fditore, pp. 373-367.

LABEL.LE, J. (I988): “Lexiques-grammaires comparés: formes verbales figées en français du Québec». Langages, vol, 9 ). pp. 73-97.

LABEL.1.F., J. (1992) : “l.inguistique et lexicographic du français du Québec : dépasser le cadre du mot», Actes du $X V^{\prime}$ C'ongrès infernarional des linguispes. Québec.

LABELL.E. J. (199.3): "Sur les expressions figés à un complément». Linquistica Communicatio, 1-2, Fes, Marox: Universits de Fes.

*LAFLEUR, B. (1991): Dirionmaire des focutions idomatiques francaises, Paris, Duculot.

LAFON, P. ( 1981$)$ : “Analyse lexicométrique et recherche des cooccurrencesm, Mots, $n^{\circ}$ 1, pp. 95-147.

LAFON. P. (1984): "Depouillements et statistiques en lexicométrie". Geneve-Paris, Slatkine-Champion. $\mathrm{XII-219} \mathrm{p}$.

LAFON, P. (I $(94)$ ) :Relations syntagmatiques, recherche des cooccurrences et segments répetes $m$. Traitements Informartse's de rorpus rexue'ls, CNRS-INal.F, Paris, Didier Enudition, pp. 161-177.

LAFON, P. el al $(148.5)$ : «Lexicométric et associations syntagmatiquesm. La Recherche fransaise par ordinateur en langu' ef lithiruturt. Charpentier. C. \& David. J. [édit.l. Cenève-Paris, Slatkine-Champion, pp. $59-72$.

LAINF, ('., PAVEL, S. \& BOILEAU, M. (1992): "la phraséologie, nouvelle composante de la recherche terminologique". L'Actualití terminologique, 25-3

LAINE. ( . (1993): "llac approche teminologique de la phraséologie», L'Actualité terminologique, $26-2$. pp. $14-16$

LAINÉ. C. (199.3) : locubulaire combinatoire de la (FAO mécanique. Secrétariat d État du Canada/Rint.

LAKOFF, (i. \& JOHNSON, M. (1980) : Melaphors We live By, Chicago. London.

LAKOFF, (i. \& JOHNSON. M. (1985) : Le's méfaphores dans la vie quotidienne. Paris, Minuit. 254 p.

LAPORTE, F. (1988) : "Lal Reconnaissance des expressions figées lors de l'analyse automatique", Langages. vol. $9(0), \mathrm{pp} .117 .126$.

LAIIREN, (h. \& NORDMAN, M. (1985): „Potential Fields of LSP Research: A Proposal for Corpus Selection and Methods", Mulilingua : Journal of Interlanguage Communication, Manchester. England, 4-4. Pp. $227-230$

LALRENT, M. (1978): Le prët-à-parler : essai es leviques, Saint-Laure.

I.AURIAN, J.-M. (198.3) : “Aspects multilingues d'une recherche lexicologique», Contrastes, Hors série A3, PP. 23.26

LAURIAN, J.-M. (198.3): aÜber die grüne (irenze ou la longue marche des lexies colorées". Contrastes. vol. 7. pp. 79-95.

LAZERSON, B. H. (1986): "Pattemed Words and Phrases", Verbatim : The Language Quarlerly. 12-3. Essex, pp. 4-6.

I.E BIIXIS. R. (1954) : «A propos des mots-tandem", Vie et langage, vol. 33, pp. 554-559.

I.FBLANC, B. ( $(943)$ : "L e compontement phraséologique des marques déposées". L'Actualité terminologique. 26-2, PP. 20-21.

L.E (IUERN, M. (1973) : Sémantique de la méfuphere et de la métonymie. Paris, Larousse.

L.EHRER. A. (1978): "Structures of the Lexicon and Transfer of the Meaning w, Lingua, 45-2, pp. 95-123.

L.EHRER. A. $(1990)$ ) :Polysemy. Conventionality. and the Structure of the Lexiconm. Cognitive Linguistics, 1-2. pp. $2017-246$

L.EPINFTTE: B. (1989): "Vers un dictionnaire explicatif et combinatoire bilingue : bases theoriques et élaboratuon de quelques anticles". (aheers de levicologie. 54, pp. 105-162.

L.EPINE:TIE-1.FPE:RS, B. (1985): "Séries lexicuatiséess el dictionnaires bilingues : étude de trois dictionnaires espagnol-françals", Meta, 30-3, pp. 242-255.

L.ERAT, P. (1494) : "Terminologie vs. lexicographie", in Candel, D. lédit.l, pp. 27-36.

L.ERAT, P. (1494) : "Dérivation lexicale et dérive terminologique”, Meta, 39-4, pp. 581 -588.

L.ERAT, P. (149.5) : I.es kangue's spécualiscie's, Paris, P(IF 
LERAT, P. \& BUDIN, G. (1992): "La formation à la rédaction technique assisté", TAMA '92. Vienne. TermNet, pp. 145-155

LERAT, P. \& SOURIOUX, J.-L. (1994): Dictionnatre juridique : Terminolesie du contrat (frangais-anglaisallemand). Paris, (ILF.

"LETHUILLIER, J. ( 1991 ) : “Combinutoire, terminologie et textes", Meta, .36-1

LEVI, J.N. (1978): The Sintar and Semamtics of Complex Nominals, New York, Academic Press.

LFVIN. B. (1993) : English Lerb (lasses and Alermations. A Preliminary Investigation. The Univ. of Chicago Press.

LEWICKI. R. (1993) : “Phrasematik im Ubersetzungstext als TRager des Fremdkonnotation», in Bessé. B. de lédit. J. pp. 359-366.

L.HOMME, M.C. (1992): "De la finalite conceptuelle au fonctionnement linguistique". L'Atrualité termimolegique. 25-1, pp. $21-22$

L:HOMMF, M.C. (1993): ales unités phraséologiques verbales et leur représentation en terminographie", in Bessé, B. de lédit.]. pp. 493-5014.

L'HOMME. M.C. (199.3) : "l ee verhe en terminologie : du concept au contexte". L'Ac'rualité terminologique. 26-2. pp. $17-19$.

LIANG, S.Q. (1991): "A propos du dictionnaire français-chinois des collocations françaises", (ahiers de levicologie, n०59, pp. 151-167.

LIEB. H.-H. ( 1992$)$ : "The General Valency Hypothesis", Actes du Xl' ('ongres internatuonal des linguistes. Québec.

LIEBERT. W.-A. ( 1994$)$ : "Lascaux - A Hypermedia Lexicon of Metaphor Models for Scientific Imagination». in Martin. W. el al. leds]. pp. 494-5(x).

LIPKA. L. (1971): "(irammatical Categories, Lexical Items and Word-Formation", Foundarions of Language. 7-2, pp. $211-238$.

LIPSHIT/. E. (1981): "La nature sémanto-structurelle des phraséologismes analytiques verbaux", (ahiers do levicologice, $38-1$, pp. $35-44$

LOEWENBERG; I. (1975) : aIdentifying Metaphorsm, Fomudations of Language. 12, pp. 315-338

LÖFFLER-1.ALIRIAN, A.-M. "' al. (1979): "Pour une Étude contrastive des lexies complexes : cas particulier des texies à chiffres en franf̧ais, portugais el finnois". ('ahiers de levicologic', 34-1. pp. 61-86.

LÖFFLER-LAURIAN, A. M. (1982) : "l, analyse contrastive des lexies complexes : questions liees aux expressions dites idiomatiques, cas des lexies avec pied et main", (ontrastes, 4-5, pp. 119-138.

LÖFFLER-LAURIAN, A.-M. (198.3) : "Fonctionnement des lexies complexes: cis des lexies contenant des noms de couleur en finnois et en françals", (imtrasle's, vol. 6. pp. 51-6K.

LÜDI, (i. ( 984$)$ : "Aspects enonciatifs el fonctionnels de la néologie lexicale". Re'herches en pragmarémannique, Kleiber. (i. lédit. I, Paris / Met/., pp. 16.5-18.3.

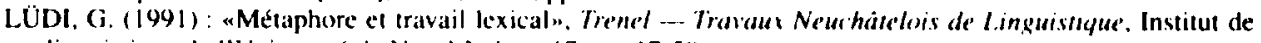
linguistique de $1^{*}$ Iniversité de Neuchâtel, no 17. pp. 17-50.

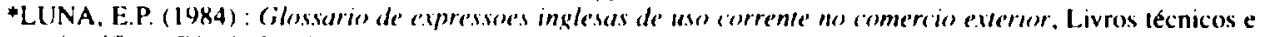
cientificos, Rio de Janeiro.

LURATI, (). (198.5): “La locution entre métaphore et histoire", La locution. Artes du rollonue international, Montreal. Universite Mc(jill, 15-16 octobre 1984. Le Moven Francas, 14-15, pp. 82-102.

MACKIN, R. (1978) : "On Colloxations: Words shall be known by the company they keep". Honour of A.S. Mornhy. Sirevens. P. /ed.]. Oxford Univ. Press., pp. 149-165.

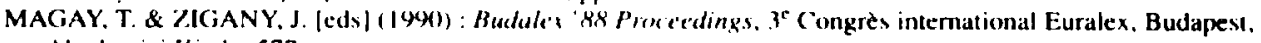
Akademiai Kiado, $577 p$

MAKKAI, A. (1972): Idiom Siructure in Engltsh. The Hague, Mouton, 371 p.

MAKKAI, A. (197.5): "The Metaphorical Origins of Idiomaticity", Four Essass on the Metaphor, Georgeton'n Werking Papers in Lingustics, Di Pietro (ed). vol. 11. pp. 10-59.

MAKKAI, A. (1978): "Idiomaticity as a language universal", Universals of Human Language, vol. 3, pp. $401-448$.

MAKKAI, A, (1987) : «Idiomaticity and Phraseology in Post-Chomskian Linguistics: The ('oming-of-Age of Semantics heyond the Sentence'. Semiofica, 64-1/2, pp. 171-187.

MAl.IS, L. (1094) : "Paradigme de la valence verbale et réalisations nominales el pronominales", Infernafional Jesurnal of l.evicographi, 7-2, pp. 142-157

MALKIEL. Y. (1959) : "Studies in irreversible binominals". Lingua. vol. 8. pp. 11.3-1(x)

MAN. O. (19.53): "Ustalena spx)jenı a tra/eologicke jednotky" ((iroupes figés et unités phraséologiques), Le'vikogrofickl shornic. Bratislava, pp. $101-110$.

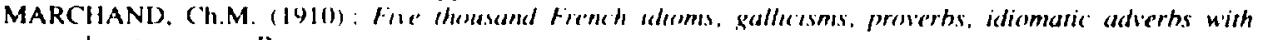
CXplandtory notes, Paris.

MARKS. (i.A. \& JOHNSON, (h.B. (1975): The mew English-French dictionary of slang and rollonquialisms. Dutton \& Co. Inc 
MAROUZZEAL, J. (1451): “Composés à l'état naissant". Melange's de' linguistique offerts d Albert Dauzat, Paris. Edition, d'Arrey, pp. $201-207$.

MAROLZI:AU, J. (1950) : “Le mot el la formule". Aspe'cts du fransais, Paris, Masson et Cie, pp. $181-197$.

MARTIN. E. ( 1992$)$ : Rocommatsance de contextes themallumes dans un corpus textuel, CNRS-INaLF, Paris, Didier Eruditıon, $283 \mathrm{p}$.

MARTIN. 1. (|994): "Theme et texte". Me'(s, 39-4, pp. 790-797.

MARTIN, J. (|984) : "al L Langue de spécialité : Propositions pour une recherchem. Bulletin de la Commission Interumberstaure Suisse de Linguisfique Appliquée. Neuchâtel. Suisse, vol. 39. pp. 23-32.

MARTIN, R. (1976): Infirence, antomymie 'l paraphrase'. Elements pour une theorie sémantique, Paris, Klıncksteck. $176 \mathrm{p}$

MARTIN. W, (1993) : aRemarks on Collocations in Sublanguages". Terminologie ef traduction, $2 / 3$, Pp. $1.57 \cdot 164$

MARTIN, W. (1994): «Knowledge-Representation Schemata and dictionary definitionsw, Perspecrives on English Sudie's in Hemor of Professon timmo borlat

MARTIN, W. e'f al leds| (1994): EURALEX 94 Proceding.. Amsterdam, $628 \mathrm{p}$.

MATORE. (i. (1962) : l. Fispace humamn. Paris. Éditıons du Vieux Colombier.

MCARTHIIR. T. (1989): “The Long-Neglected Phrasal Verh". English Today : The International Review of the English Languagr. Cambridge. England. Apr. : \$-2(18), pp. 38-44.

MCNEII.L. D. (196.3): "The origin of associations within the same grammatical class», Journal of Verbal Le'arning and lerbal Bcharior, vol. 2, pp. 250)-262

MELLČClK. I. ef al. (1981): al In nouveau type de dictionnaire : le Dictionnaire explicatif et combinatoire du franfals contemporain”, (ahers de lenicelogie, Paris, 38. pp. 3-43.

MEL'CUK, I. et al. (1984) : Dictionnaire explicasif of combunatorre du francais contemporain. Recherches lexico-reimantiques I. (DECF("). Montréal. Presses de I’Université de Montréal, $172 \mathrm{p}$

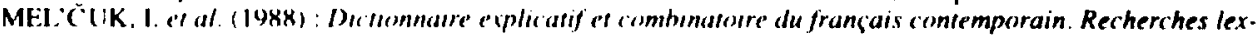
ior)-semantiques /I, (D)ECFC), Montréal, PUM

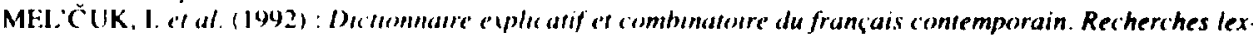
ioro-semantiques III, (DECFC). Montréal, PUIM, $323 \mathrm{p}$.

MEL'CLUK, 1. \& WANNER. L. (1994): "Toward and Efficient Representation of Restricted Lexical Coxecurrence", in Martin. W. el al. [eds|, pp. 325-228

MFPHAM, M. (1970) : "l.'Ordinateur et l'analysc grammaticale", les langues de spécialité : analyse linguisrique "f recherche pedagogique Acte's du stage de Saint-C\%od, 23-30 novembre 1967. Strasbourg. AIIIII.A. pP. $1+1-150$.

MEYER. I. \& MACKINTOSH. K. (1994) : "Phraseme Analysis and Concept Analysis: Exploring a Symbiotic Relationship in the Specialized Lexicon ", in Martin. W. "al leds]. pp. 339.348.

MILLER. (;. $(\mid \varphi(M))$ : "Nouns in WordNet: A Lexical Inheritance System", International Journal of Leweography, 3-4, pp. 245-265

MIL.NER. ( i.B. ( 1964$)$ : "De l'amature des locutions proverbiales. Essai de taxonomic semantique». $L H o m m e$. 9.3. pp. 49-70.

MISRI, (i, (1987): "Approches du figement linguistique : critères et tendances», Linguistique (La), $23-2$. pp. $71-8.5$

MOESCHLER, J. (1993) : “Idiomes et locutions verbales», in Besse, B. de ledit.], pp. 1.35-148.

MOIGNET, (i. (1974): "Liadverbe dans la locution verbale". Eiludes de psycho-systématique française. (Bibliothèque française et romane. Série A. Mamuels ef études linquistiques, vol. 28), Paris, Klincksieck. pp. 137.1 .59

MOLHO, M.. HLANCHE-BENVENISTE. C.. CHERVEL. A. \& GROSS. M. lédit.| (1988) : «L'Hypothèse du formant sur la constitution du signifiant: Esp. un / nom. (irammaire el histoire de la grammaire. Aix-enProvence. Univ. de Provence. 494 p.

MOLINO. J. (1979) : "Métaphores, modèles et analogies dans les sciences m, Langages, vol. 54, pp. 83-102.

MOL.INO. J.. SOUHL.IN. F. \& TAMINE, J. (1979): "Problemes de la métaphore", Langages, 54, Didier, I arousse, pp. 5-40.

MORF:AU, M.L. ( 4986$)$ : «Les séquences preformées : entre les combinaisons libres et les idiomatiques. Le cas de la négation avec ou sans ne", Le fransais moderne. 54-3/4, pp. 137-160.

MORIN, Y.-C. (1975) : «A Remark About Lexicalization of Syntactic Expressions», Recherches linguistiques, Montréal. vol. 4. p. 173.

MORTURE:UX. M.F. (198X) : "L'ambiguité dans les paraphrases de termes scientifiques», L'ambiguiré ef la paraphrase. Univ de Caen, pp. 293-298

MORTURE: $X, M, F,(1994)$ : "L, analyse du discours de la vulgarisation scientifique et le dictionnaire de la langue scientifique", in ('andel, D. lédit.l. pp. 6.3-75

MOSKAL,SKAJA, ().J. (1975): «Fixed Word Combinations of Serial Formations as Objects of Grammars, Linguistus, vol. 143, pp. 44-59. 
MOUNIN. G. (1964): "Les groupes de mots - Les formes idiomatiques", la machine à traduire, (Janua Linguarum, Series Minor, vol. 32), La Haye, Mouton, pp. 1,32-14.5.

MROSOWSKI, B. \& COUI.ON, P. (1978) : "Quelques aspects de la phrasélogie comparé", Fremdsprachen. 22-2. pp. 125-12X.

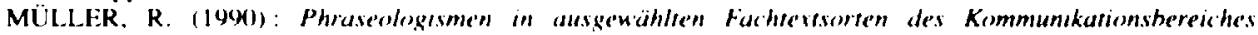
Humannu'dizin im Enghsshen. (Dissertation). leiprig.

*NASCENTES. A. (1987): Tesaum da fraseologia hrasilerira. Nova Fronteira. Rio de Janeiro.

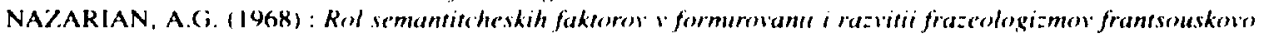
iazıka (Le rôle des facteurs sémantiques dans la formation et le développement des phraséologismes de la langue françaisel, Moscou.

NAZARIAN, A.6. (1976): Frazcologia sovremennovo framlsouskovo iazka (Phraséologie du français contemporain]. Moscou. Vischaia Chkola.

NEDOBITY, W. $(199())$ : "Simple phrase structure grammars and their application in terminology".

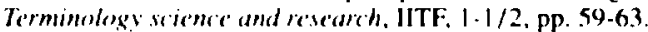

NEUHAIS, J.-P. (1993) : “l a phrasécologie dans l'entreprise”, in Bessé. B. de lédit.l. pp. 237-244.

NEWMARK. P. $(|(\mathcal{x})|)$ : "Paragraphs on Translation", The Linguist, 30-1, pp. 28-32.

NORMAND, C. (1976): Méfaphore' et come ept. Paris, PUF

NORRICK. N. (1981) : Semiotic Primeiples in Semantic Theory. Amsterdam, John Benjamins

OPIT/, K. (1983): "Linguistics between Artiticiality and Art: Walking the Tightrope of L.SP Research". Bulletin ( $/ L A$, vol. 37 , pp. 8-20.

OPIT/. K. (1492): "On the Borders of Semantic Invariance: Connotation and the Dictionary", Euralex 92 Procecedens.s, Tampere, pp. 398-403.3.

*ORNE. J. (1921): The Language of the Foreign Book Trade: Abbrevianons. Terms and Phrases. len 10 langues). Rivista bimestre cubana.

ORR. J. (1962) : Old French and modern English idiom.s, Oxford. Blackwell.

OSTRA. R. (1985) : "La Perspective fonctionnelle de la phrase en tcheque et en françaism, Éludes Romones de Brno, vol. 16, pp. 7-IS

PADUCHEVA. E.V. \& RAKHILINA. E.V. (1993) : "Predicting Co-occurrence Restrictions by Using Semantic Classifications in the Lexicon". Voprosy jazbkoznanija 6. Moscou.

PARC, F. (1993): “Phraséologie termınologique dans les textes législatifs et réglementaires», in Bessé. B. de [édit.l, pp. 219-236.

PAVEL, S. (1989): Dicrionmore francais-anglais de I'Infelligence logicielle. Otlawa. Secrétariat d'État du Canuda/Rint, $50.3 \mathrm{p}$.

PAVEL, S. (1991): "TERMIUM : Le eraitement informatisé des difficultés de traduction". Actes du Colloque

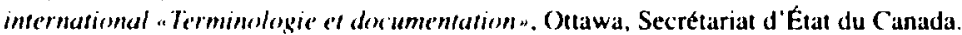

PAVEL, S. (199.3) : "Vers une méthode de recherche phrasécologique en langue de specialite", L'Actualité rermi. nologique. 26-2. pp. 9-13.

PAVEL. S. (1993): "Neology and Phraseology as Terminology-in-the-Making". Terminology : Applicarions in Interdisiplinary Communication. Sonneveld. H.. Loening. K. [eds]. Amsterdam. John Benjamins. pp. $21-34$.

PAVEL. S. (199.3): "la phraséclogie en langue de specialite". Terminologies nouvelles. 10. Bruxelles. Rint, pp. $67-82$.

PAVEL. S. \& BOILEAL, M. (1994): Vocubulaire de's systemes dynumiques ef de l'imagerie fractale, Otawa, Rint, $217 p$

PAVI OVITCHOVA-PROUJINTSOVA, M. (1978): „Pohlad wovietskih frazeologov na poxtstatou frazeologitskei iednotki a klasifikatsiou frantsouskih frazeologitcheskih iednotiek" |Les idées des phraséologues soviétiques sur l'essence de l'unité phrasécologique el sur la classification des unites phraséologiques du françaisl. Tsizl laziki in (hkole, Prague, vol. 22, pp. 409-420.

PAWLEY. A. (198.5): "On speech formulas and linguistic competence", Lenguas Mrdernas, CHL, $\mathrm{n}^{\circ} 12$. pp. 84-114.

PERMJAKOV. G.L. et al. (1984): “On Paremiological Homonymy and Synonymy". Kodikas/Code/Ars semeri ofica. D -7400 Tübingen, 7-3/4, pp. 269-271

PERREAI, R.A. \& I.ANGFORD. M.J. (1972) : "oncise Fench-American dicfionary of figurative and idiomatic Language. Lexique anéricain-francais de las langue daiomatique, Parss, Ophrys.

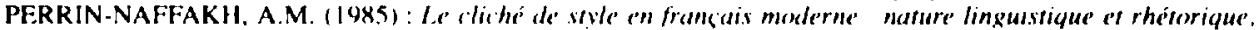
foncrion listriraire, Lille.

PESANT, (i. \& THIBALIL, E. (199.3) : “Termınologie et cosccurrence en langue du droit". Terminologics notevelles, 10, Bruxelles, Rint, pp. 27-35.

PHAL. A. $(1964)$ : "Les groupes de mots et les problèmes qu'ils posent dans la préédition de textes scientifiques destinés a l'analyse mécanographique", (ahiers de levicolegice. 4-1. pp. 45-60. 
PHILLIPS, M.K. (1981) : “Toward a Theory of LSP Methodology», Languages for Specific Purposes: Program Design and Evaluation, Rowley, MA, Newbury House, pp. 92-105.

PIACENTINI, J.A. (1981): "La création des synthèmes publicitaires et leur integration dans le langage courant ", La Linguistique'. 17-1, pp. 49-76.

PICHT. H. (1987) : "Terms and their LSP Environment - LSP Phraseology», Meta. 32-2, pp. 149-155.

PICHT, H. (1989) : «Fachsprachliche Phraseologien. Special Language: From Human Thinking to Thinking Machenes. Papers presented at the 6th European Symposium on LSP, University of Vaasa. Lauren, C. \& Nordman. M. |eds]. Clevedon. Philadelphia, pp. 89-i(19).

PICHT. H. (1990) : aLSP Phraseology from the Terminological Point of Views. Terminology Science and Research, IITF, 1-1/2, pp. 33-48.

PICHT, H. $(\mid \varphi 90)$ : "A Study of L.SP Phraseological Elements in Spanish Technical Texts», Terminology Science and Ressarch. IITF, 1-1/2.pp. 49-58.

PICHT. H. (1944): "The Multidisciplinary Nature of Terminology: Remembering Eugen Wuster», in Kocourek. R. |ed|, pp. I.37-|6|.

PICOCHE. J. (198.5) : “Un essai de lexicologie guillaumienne : la locution figée comme revelateur du signifie de puissance des polysèmes". La lecution. Actes du colloque infernational, Montreal, 15-16 octobre 1984, Le Moven Fransais, vol. 14-15. pp. 103-118.

PICOCHE, J. (1986) : Structure's sémantiques du kerique français, Paris, Nathan, 144 p.

PIGUET. M.-F. (1994): "Élémenıs sur la formalion d'un vocabulaire specialisé: l'economie politique», in Candel, D. [edit. ., pp. 137-150.

PILZ., K.D. ( $|98|$ ) : Phraseologie: Redensarlenforsschung. Stuttgan.

PINEIRA-TRESMONTANT, C. (1988) : “Rigidités discursives et flou sémantique. La notion de Lexle», Mots, $n^{\circ}$ 17.pp. 145-169

PIOT. M. (1988) : "Conjonctions de subordination et figement", Langages. vol. 90, pp. $39-56$

POLINSKY, M. (1992): "Beyond the NP: the Sentence as Media of Reference". Actes du XVe Congres international de's linguistes. Quebtec.

POPLACK. S. (1988): "Conséquences linguistiques du contact des langues; un modele d'analyse variationnistem, Langage et Sinciéts, 43, pp. 23-48.

POTTIER, B. (19621 : Systématiqué des éléments de relation. Paris, Klincksieck.

POTTIER, B. (1964): "Vers une sémantique modemen. Travaux de linguistique el de listérature, publit par le Centre de Philologie et de L.itteratures Romanes de l'Université de Strasbourg, 2-1, pp. 107-137.

POTTIE:R, B. (1974): Linguistique générale : théorie e's description. Coll. Initiation a la linguistique, Strie B, Problemes et meithesdes, vol. 3. Paris, Klincksieck, $33.3 \mathrm{p}$.

PRUVOST. J. (1994): "L'illustration dictionnairique et les technolectes dans les dictionnaires semasiologiquesm, Mera, 34-4, pp. 741-756.

PUSTEJOVSKY. J. [ed] (199.3): Semantics and the Lexicon. Kluwer, Dordrecht.

PUSTEJOVSKY, J. (199.3): "Type Coercion and Lexical Selection». Semantics and the Lexicon, Kluwer. Dordrecht, pp. 73-94.

QUEMADA, B. (1978) : "Technique et langage”, Histoire des lechniques. Paris. Gallimard, pp. 1146-1240.

RASMUSSEN, J. (1981): "Analyse de texies en langues de specialité, Études françaises en Europe non francoptrone. Warsaw-Wroclaw. Panstwowe Wydawnictwo Naukowe. Uniwersytet Wroclawski, pp. 88-94.

RASTIER. F. (1987) : Sémantiqué interprétative. Paris. PUF. 277 p.

RAT, M. (1957) : “Deformations populaires des mots et locutions», Vie el Langage, vol. 59, pp. 89-93.

RAYMOND, M. ( $(942)$ : "Bibliographic phraséologique". Dictiemnaire explicatif et combinatoire du français contemporain. Recherrhes lecuco-simumuques III. Mel'cuk, I. es al., Montreal, PUM, pp. 81-92.

REBOUL, O. (1979) : “Le slogan et les fonctions du langage", Le français dans le monde, vol. 143, pp. $21-26$.

REDARD, F. W WLER, S. |eds| (198,3): Langues de spécialite : Thésrie, application el enseignement, Actes du colloxque de la Commıssion interuniversstaire suisse de linguistique appliqué. Bulletin de la Commission Interunurerstuarre Suisser de Linguissique Appliquéc. Neuchátel. Suisse, vol. 39.

REICHL.IN(i, A. (1993) : "le traitement de la phrasécologie dans EURODICAUTOM», in Bessé, B. de [édit.], pp. $4 \times 5.492$.

REINAL, R. (1993) : «le défi phraséologique : stratégies lexicographiques et terminologiques», in Bessé. B. de lédit.|, pp. 439-446.

REY. A. (1973) : “La phraseologie et won image dans les dictionnaires de l'age classiquew. Travaux de linguistiques et de littérature, 11-1, pp. 97-107.

REY. A. (1976) : «Structure semantique des locutions françaises". Actes du XII/ congres international de lingussricur et de philologie romanes. Quebec. PUL., vol. 1, pp. 831-842.

REY, A. (1977): "Les limites du lexique», Le lexique" : images er modeles, Paris, A. Colin.

REY. A. (1985): "Les implications theoriques d'un dictionnaire phraséologique». Le Moyen Français, Montréal, Canada. 14-15. pp. 119-13.3.

REY. A. (1987) : “Un dynamisme ambigu : le français". Qui Vive International. $n^{\circ}$ 5, pp. 72-73. 
REY. A. (1992): «BUDALEX Presidential Debate 1988». International Journal of Lexicography. Oxford University Press. 5-4.

REY. A. (1993) : «Traduire, interpréter : les mots pour le dire", in Bessé. B. de [édit.]. pp. 13-24.

REY. A. \& (HANTREAU, S. (1993) : Dic tomnaire des expressions at locutions figurées. Paris. Coll. Les Usuels du Rober, $888 \mathrm{p}$.

RIABTSEVA, N.K. (1993): «Metadiscourse Collocations in Scientific Texts and Translation Problems: Conceptual Analysis», in Bessé, B. de lédit. I. pp. 375-386

RICHARD, G. \& BÉRARD-DUGOURD, A. (1986) : «Le traitement des tocutions dans l'analyse du langage naturel", Étude n 101 du Centre scientifique IBM France.

RICCEUR, P. (1975) : La Méraphore Vive. Paris. Seuil. 414 p.

RIEGEL. M. (1989): "Avoir + attribut de l'objet: construction syntaxique et paradigme idiomatique». Europhras 88. Phraséslegie Contrassive. Actes du colloque international. Klingenthal-Strasbourg 12-16. mai 1988. Gentud Gréciano, [édit.]. Récherches (iermaniques. Strasbourg, vol. 2, pp. 337-347.

RIFFATERRE. M. (1964) : "Fonctions du cliché dans la prose littéraire", Cahiers de l'Assesciation interna sionale de's études française's, vol. $16, \mathrm{pp} .81-95$.

RIGAUD, L. (1881) : Dictionnaure des lieux communs, Paris, Paul Ollendorf, 327 p.

RIGOL.OT. F. (1985): “Perspectives ré́toriques et sémiotiques sur la locution : Locutio/Location, Le Moven Français. Montréal, 14-15, pp. 4(X)-418.

ROBACK. A.A. (1979): A Dictiomary of Imernational Slurs, with a Supplementary Essay on Imternational Prejudice. Waukesha. Maledicta Press, 394 p.

ROBERTS. R.P. (1992): "Identifying the Phraseology of Languages for Special Purposesm. Actes du XVe Congrès international de's linguistes, Quétec.

ROBERTS, R.P. (1993) : "Phraseology : The State of the Art", L Actualite terminologique, 26-2. pp. 4-7.

ROBERTS, R.P. (199.3) : “La phrasécologie : état des recherches», Terminologies nouvelles, 10, Bruxelles, Rint, pp. 36-42.

ROBERTS, R.P. \& SIMARD. C. ( 1980$)$ : "Le's unités verbe/substantif dans la langue économique», Langues de spécialité, vol. I. pp. 41-70.

RODEGEM, F. (1972) : “Un problème de terminologie : les locutions sentencieuses". Cahiers de l'Institut de linguistique (Louncan), 1-5. pp. 677-70)3.

*ROEGIEST, E. \& TASMOWSKI. L. |édit.] (1983) : Verhes et phrases dans les langues romanes : Mélanges offerts a Louis Mourin, $291 \mathrm{p}$

ROGERS, D. (1474): Less verhes a la fois transitifs et intransitifs en français contemporain. The Hague, Mouton, $334 \mathrm{p}$.

ROGIVUE, E. (1965) : Le Musíe des gallicisme's, Genève, Georg.

ROHRER, (h. (1967) : «Definition of locutions verbales», The french Revien', 41-3, pp. 357-367.

ROLAND. H. \& BOYER. L. ( 1983 ) : Dictionnaire des expressions juridiques, Lyon, L'Hermes.

RONSSE, F. (1963) : "Locutions françaises issues de mots russes uniques". La traduction automatique, vol. 1. pp. 10-21.

ROSSENBECK, K. (1989) : «Lexikologische und lexikographische Probleme Fachsprachlicher Phraseologie aus kontrastiver Sicht". Transtation and Lexicography. Papers read at the EURALEX Colloquium Innsbrack, 2-5 Juillet 1987, Kirksville. Missouri, Snell-Hornby. M. et al leds]. pp. 197-210

ROUGET, Ch. (1994): "Comment rendre comple des locutions verbales?". International Journal of Lexicography, 7-2, pp. 177-196.

ROULET, E. (1978) : „Essai de classement syntaxique et sémantique des vertes potentiellement performatifs en français". C'ahiers de linguistiques. vol. 8. pp. 437-455.

ROUSSEAU, L.-J. (1993): "Terminologie et phraseologie, deux composantes indissociables des langues de specialité", Terminolongies nouvelles, 10, Bruxelles, Rint, pp. 9-12.

ROVENTA-FRUMUSANI, D. (1989): "Cognitif et expressif dans l'élude de la métaphore", Revue Roumaine de Linguisrique, 34-6, pp. 52.3-529.

RUNDELL, M. \& STOCK, P. (1992) : “The Corpus Revolution», English Today, 30, pp. 9-14.

RUWET, N. (1990) : “Des expressions metétorologiques», le francais moderne, 58-1/2, pp. 43-97.

SAGER. J.-(. (199.3): "Future Developments and Research in Phraseology and Terminology related to Translationm, in Bessé, B. de lédit.], pp. 58.3-588.

SAINÉAN, I.. (IM)S) : "la création métaphorique», Beihefie zur Zeisschriff für romanische Philologie, Halle. $1-10,1907$

SALEM. A. (1987) : Pratıque des segments répétés, Essai de statistıque textuelle, Paris, Klincksieck, 335 p.

SANDMANN, M. (1973) : "Monter a cheval et le problème des variations d'expressions conditionnees par les contextes situationnelsm. Expériences ef critiques, Bibliothèque française et romane, Strie A, vol. 25, Paris, pp. 221.248.

SANFILIPPO, A. (1992): "Verbal Diatheses: Knowledge Acquisition, Lexicon Construction and Dictionary Compilation", Acquilex-Il working paper. 
"SARMIENTO. F. (1970): Dicrionarie) de voces y frases gallexas, 12 volumes, Universidad de Salamanca.

SAUVAGEOT, A. (1955) : wLes mots-tandem ", lie ef Langage, 34-35, pp. 223-226.

SAUVAGEOT. A. (1964): Perrrait du vocabulaire fronsais, Paris. Larousse.

SCHAFT/EN, C. de (199.3): "Un acces rapide aux collocations". Terminologies nowvelles, 10. Bruxelles. Pp. 103.115 .

SCHINDLE:R, W. ( $(493)$ : "Phraseologismen und Wortfeldtheorie". Studien zur Worffeldtheorie, Tubingen, Niemeyer.

SCHMID. A. (I989): "Wöterhucher als Hilfe sur Übersetzung von Phraseologismen", Translation and Lexicograph: furalex 1987. Snell-Homby, M.. Pb̆hl. E., Bennani. B. [edit.]. Amsterdam, John Benjamins, pp. $121-127$

SCHMID. A. (1984): tin Beitrag zur Phraseologie des Französıschen. Syntaktisch-semantische Untersuchungen iu mettre in festen V'rbindungen. Innsbruck. Verlag des Instituts für Sprachwissenschafi der I Iniversit tat Innsbruck. (Innsbrucker Beitrige eur Kulturwissenschaft. Sonderheft 57).

SCHMID. A. (1943) : "Phraseologismen - Crux der Maschinenubersetzung*, in Bessé. B. de [Edit.].

SCHNEIDFR, F. ( 1989$)$ : "Comment decrire les actes de langage? de la linguistique pragmatique a la lexicographie : Ia belle affaire ! el Tu m'en diras tant !", linguisfıche Arbeiten 227, Tubingen, Niemeyer, 532 p.

SCHUR, N, (1986): “Misquotationsw, Verbatim : The Language Quarterly, Essex, 13-1, pp. 12-13.

SECHEHAYE. A. (1921); "I axutıons et composés". Journal de psychologie normale ef pathologique, vol. 18. pp. 6.54-67.5

SELLE, S. (198.5): "Etudes de la valence des verhes comme méthoxle de recherches pour le français de spécialitém, Spectal Language / Fachsprache, A-1230 Vienne, 7-3/4, pp. 136-143.

SERANT, D. \& THOIRON, Ph. (1988): „Topographie des formes répétées $*$ Informatique et Statistique dans les Sicrences /lumaines, 1-4. pp. 3.3.3-34.3

SERGIJEIEV, V.N. (1964) : “Bibliograticeskỹ ukuzatel literatury po frazeologii izdannoj v SSSR s 1918 po $1969 \%$. (Bibliographice phraséologique des ouvrages publiés en URSS entre 1918 et 1961, domaine du français). Problemi frazeologui |Problèmes de la phraséologie]. Babkine. A.M. [édit.]. Moscou-Leningrad, Akademia Naouk SSSR, Institut Rouskovo Jazika. pp. 255-309.

SIALM, A. (1988): Semiotih und Phraseologle'. Zur Theorie fester Wortferbindunger im Russischen, Publications Universitaires Europénnes, Série XVI, vol. 37. Peter Lang. Beme.

SIMATOS, I. (1988) : Elémenrs pour une thérme de's evpessions idiomatiques : identite lexicale, reférence et relanions argumu'nrule's. Theve Paris VII, 1987. L.ille. Atelier national de reproduction des theses.

SINCLAIR. J.M. (I98()): “Some Implicatıons of Discourse Analysis for ESP Methodology», Applied Linguistucs, Minneapolis. MN. 1-3. pp. 253-26I.

SINCLAIR, J.M. (1988) : “Collocation : a progress report», Language Topics (in honour of M. Halliday), Steel, R. el al. [eds], Amsterdam-Philadelphia, vol. 2, pp. 319-331.

SINCLAIR, J.M. ( 1991$)$ : Compus. Concordance. Collocalion. Oxford Univ. Press, Oxford.

SKORUPKA, S. (1966): "Ce que lion entend par idiomatisme", Slavica, Debrecen (Hongrie), vol. 6. Pp. $16.3-166$.

SLOBIN, D. (1991): “leaming to think for speaking: native language, cognition and rhetorical stylen, Pragmarics. 1. pp. 7-25.

SMADJA, F. (1993): «Retrieving Collocations from Text: Xtract», Compurational Linguistics, 19-1, pp. 14.3-177

SMADJA, F. \& MCKEOWN. K. (1991): "Using Collocations for Language Generation*, Computational Intellige'nic, 17

SOKOLOVA. (i.G. (1987) : «On the formation of phraseological units in French», Voprosi lazikoznania, vol. 3. pp. $91-101$.

SOMERS, H.L. (1987): Valency and Case in Computarional Linguistics, Edinburg.

SONNEVELD. H.B. \& LOENIN(i. K.I. leds] (199.3): Terminology. Applications in Interdisciplinary Communicution. John Benjamins Publ. Co. Amsterdam. $244 \mathrm{p}$.

SORENSEN, K. (1986) : "Phrasal Verb into Noun”. Neuphilologische Mirteilungen, 87-2. pp. 272-283.

SOWA, J.F. (1984) : Conceptual Structures, Reading MA, Addison-Wesley, 481 p.

SPENCE. N.C.W. (1969) : aComposé nominal. locution et syntagme libre», La linguistique. 5-2. pp. 5-26.

SPERBER, D. \& WILSON, D. (1986) : Relevance. (ommunication and (ognition, Ox ford, Basil Blackwell.

SPILLNER, B. (1982) : „Pour une analyse syntaxique et stylistique des langues françaises de spécialité, Les Langues modernes. 76-1. pp. 19-27.

SPILLNER, B. (1994) : "Terminologie el connotations", in Candel, D. [édit.], pp. 53-62.

SPITZIER, L. (1948) : "Courir la calabre". Le francais moderne. vol. 16, pp. 165-166.

STADLER, 1.G. de (1992): "Syntagmatic lexical relations: a lexicographical perspective», Euralex '92 Procedings, Tampere, pp. $411-418$.

STEFANO, G. di \& MC (ILLLIVRAY, G. (1985) : "La locution", Actes du colloque international La Locution, Montreal. 1984, Le Meven Françuis, 14-15, pp. 3-18. 
STEFANOVA. L. \& PRIMATOROVA-MILCEVA, A. (1985): "À propos de quelques especes d'unites phrastologiques terminologiques dans la langue française et leurs correspondances fonctionnelles dans les langues allemande et bulgarem. Sapostaviteino ezikoznanie, vol. 2, pp. 8-17.

SWEETSER, E. (1992): "Metaphor, Myth and Everyday Idiom". Actes du XVe Congres international des linguistes, Québec.

TAGNIN, S.E.O. (1993) : “What's in a verbal colligation? Project for a bilingual dictionary of verbal colligations English-Portuguese/Englishm. in Bessé. B. lédit.]. pp. 149-156.

TALLGREN-TUULIO. O.J. (19.32) : "Locutions figurées calquees et non calquées. Essai de classification pour une série de langues litteraires". Mémoires de la Société Nés-philologique de Helsinki, vol. 9, pp. 279-324.

TALMY, L. (1985) : “Lexicalization Patterns: Semantic Structure in Lexical Forms». Language Typology and Syntactic Description, vol. 3. Cambridge, Cambridge univ. Press, pp. 57-149.

TAMBA, I. (1983): "La Composante reférentielle dans un manteau de laine, un manteau en laine», Langue Franşaise. Paris, vol. 57. pp. 119-128.

TAMBA-MECZ, 1. (1981): Le sens figuré. Vers une théorie de l'énonciation figurative. Coll. Linguistique nouvelle. Paris, PUF.

TELIYA. V. (1992): "The motivational basis in the semantics of idioms and ways of its presentation in the computer data base". Euralex ' 92 Proceedings, Tampere, pp. 433-439.

TELIYA. V. (1994) : «Lexical Collocation: Denominative and Cognitive Aspects», in Martin. W. ef al. [eds], pp. 368-377.

TER-MINASOVA, S. (1992): "The Freedom of Word-Combinations and the Compilation of Leamers" Combinatory Dictionary», Euralex '92 Proceedings. Tampere. pp. 533-539.

TESNIËRE, L. (1959) : Éléments de syntaxe structurale, Paris, Klicksieck.

THAGARD, P. (1992) : Conceptual Revolutions, Princeton, New Jersey, Princeton University Press.

THAGARD. P. ef al. (1990): "Analog Retrieval by Constraint Satisfaction», Artificial Intelligence. 46. pp. $259-310$.

THELEN, M. (1992) : «Lexical systems and lexical domains as measures of accessibility, consistency and efficiency of lexical information in dictionaries», Euralex ' 92 Proceedings, Tampere, pp. $441-448$.

THIELE, J. (1980): «Zum franzosischen nominativen Phraseologismus : Versuch einer Klassifizierung nach semantischen Kriterienw, Linguissische Arbeitsherichte, vol. 26, pp. 65-71.

THIELE. J. (1988) : "Les Phraséologismes dans les dictionnaires bilingues», Wissenschaftliche Zeitschrift der Einst-Morit:-Arndt-Universitaat Greifsw'ald, 37-2, pp. 49-51.

THIELE. J. (1990) : «Phraseologie = Phraséologie», Lexikon der romanistischen Linguistik Französisch. 5-1. Tübingen. Niemeyer, pp. 88-94.

THOIRON. Ph. \& BEJOINT. H. (1989) : "Pour un index évolutif el cumulatif de cooccurrents en langue techno-scientifique sectoriellem, Mera, 34-4, pp. 661-671.

THOIRON, Ph. (1993): "L'analyse quantitative des textes scientifiques», in Amaud. P. \& Thoiron. Ph. [édit.]. pp. $133-146$.

THOIRON, Ph. (1994): "La terminologie multilingue : une aide a la maitrise des concepts», Meta, 39-4, pp. 76.5-773.

THOMAS. P. (1993) : “Choosing Headwords from LSP Collocutions for Entry into a Terminology Data Bank». in Sonneveld, H. \& Loening, $K$. |eds], pp. 43-67.

THUN, H. (1975) : "Quelques relations systématiques entre groupements de mots figés», Cahiers de lexicologie. 27-2, pp. 52-71.

THUN, H. (1978): Prohleme der Phraseologie. Untersuchungen zur wiederholten Rede mit Beispielen aus dem Französischen, Italienischen. Spanischen und Rumänischen. (Beihefte zur Zeitschrift für romanische Philologie 168). Tübingen.

TOSSAVAINEN. L. (1492): "Zur Rolle der Phraseologismen bei der Nomination". Neuphilologische Misteilungen, vol. 4.3, pp. 75-86.

TOURNIER. J. (1985): Introduction descriptive a la lexicogénétique de langlais contemporain. Paris Champion.

*TRISTA PEREZ, A.M. \& CARDENADO MORE. Z. (1986): “Elementos somaticos en las unidades fraseologicasm, Annuario L/L, no 17, pp. 55-68.

TURNER. M. (1990) : “Aspects of Invariance Hypothesis», Cognitive Linguistics, vol. 1-2, pp. 247-255.

URDANG, L. (1986) : «Language Changes». Verbatim: The Language Quarterly. Essex, 12-4.

URDANG, L. \& ABATE, F. leds] (198.3): Idioms and Phrusiex Index, Detroit, Gale Research, p. 1691.

VACHEK, J. (1994): "La langue écrite selon la perspective fonctionnaliste", in Kocourek. R. [édit.]. pp. $361-399$.

VALLETTE. C. \& BURNAM. T. (1978) : Encyelopédie des idées rę̧ues, Monaco, Éditions du Rocher.

"VANDELLI, C. (1977) : Dizuenario fraseoslogico di inglese technico. Mondinie Siccardi.

VAN DER VLIET, H. (1994): «Conceptual Semantics for Nouns", EURALEX'94 Procedings, Martin, w. et al. [eds]. Amsterdam. pp. $216-225$ 
VAN DER WOUDEN, T. (1992) : «Prolegomena to a Multilingual Description of Collocations», Euralex '92 Proceedings. Tampere, 1992, pp. 449-456.

VAN EECKE. D. (1979) : «L'expression des relations temporelles par les locutions». Zielsprache Französisch. vol. 4, p. 177

VAN VOORST, J. (1994): aThe Role of Verb Meaning in the Calculation of Aspectual Interpretationsw, in Martin, W. et al. [edsl. pp. 384-389.

VARANTOLA, K. (1992) : "Words, Terms and Translators», Euralex 92 Proceedings, Tampere, pp. 121-128.

VARANTOLA, K. (1993) : "Technical Vocabularies and Learners' Dictionaries", English Far and Wide. Annales Universitatis Turkuensis, B:197, pp. 249-260.

VERKUYL, H. (1994): "Knowledge Representation in Dictionaries», Keynote lecture, EURALEX 94, Amsterdam.

VERLINDE, S. \& BINON, J. (1994): «The Dictionnaire Contextuel du Francais Economique: A Production-Oriented Dictionary of Business Frenchx, in Martin, W. ef al. [eds], pp. 523-529.

VIAN, R. \& VIAN. M. (1969): Vecabulaire et phraséologie modernes. Nach Sachgebieten geondnet. Einführung in den aksuellen französischen Wortschatz, Vienne.

WALLACE. M.J. (1979) : «What is an idiom? An applied linguistic approach». Exeter seminar on lexicography, 1978, Dictionaries and their users, Exter linguistic studies, vol. 4. pp. 63-70.

WANDRUSZKA. M. (1979): «Kontrastive Idiomatik». Festschrift Kurt Baldinger zum 60 Geburstrag. Tübingen. Niemeyer, vol. 2. pp. 951.963.

WANNER, L. [ed] (1995) : Lexicul Functions in Lexicography and Nasural Language Processing. Amsterdam. J. Benjamins.

WARTBURG. W. von \& ZUMTHOR, P. (1958) : Précis de syntaxe du français contemporain, Berne, Francke. $2^{e}$ ed. $356 \mathrm{p}$.

WEINREICH. U. (1969): aOn the Problem of Idiomsm, Substance and Siructure of Language, Los Angeles, pp. 23-8I.

WIER7.BICKA, A. (1982): *Why Can You have a drink When You Can't have a eat?, Language, 58-4, pp. 753-799.

WILMET, M. (1986) : La détermination nominale, Paris, PUF

WOLF, B. (1988) : "Wörterbuch und Wortverknüpfung», Sprachpflege, vol. 8, pp. 112-115.

"WOOD, F.T. ( 1978) : English Prepositional Idioms, London, MacMillan, 562 p.

WOOLDRIDGE. T. (1985) : “La Locution et les premières dénominations de locution dans le métalangage dictionnairique français». Le Moyen Français. Montréal, no 14-15, pp. 437-449.

YAMADA. H. (1979): "Idioms from a New Point of View: A Trace Theoric Approach*, Festschrift for Professor Kazuko Inoue. Tokyo, pp. 529-551.

YOKOI. T. (1993) : „Knowledge Archives», in Sonneveld, H. \& Loening, K. [eds], pp. 181-193.

ZERABA, L. (1977): "Sur la méthode deelaboration des dictionnaires phrastologiques*, Terminologies, vol. 76, pp. II.25-11.35.

ZGUSTA, L. (1984): "Translational equivalence in the bilingual dictionary", LEXeter'83 Proceedings, Hartmann. R.R.K. [ed]. Tübingen. M. Niemeyer, pp. 147-154.

ZHUKOV. V.P., SIDORENKO. M.I. \& SHKLJAROV, V.Y. (1987): Slovar frazeologicheskih sinonimov russkogo jazyka, Moskva, Russkij jazyk.

ZIPF, G.K. (1932) : Selected Studies of the Principle of Relative Frequency in Language, Cambridge, Mass. Harvard Univ. Press.

ZOLONDEK, D. (1988) : “le syntagme fige dans le domaine de la telematique : approche terminologique». Langues et Linguistique, n_ 14. pp. 303-323.

ZWANENBURG, W. (1970) : "La classification des composes en français moderne», Lingua. $25-2$. pp. $128-141$.

Silvia Pavel

Bureau de la traduction. Montreal Canada 